\title{
PRINCIPAL EIGENVALUE MINIMIZATION FOR AN ELLIPTIC PROBLEM WITH INDEFINITE WEIGHT AND ROBIN BOUNDARY CONDITIONS
}

\author{
M. HINTERMÜLLER, C.-Y. KAO, AND A. LAURAIN
}

\begin{abstract}
This paper focuses on the study of a linear eigenvalue problem with indefinite weight and Robin type boundary conditions. We investigate the minimization of the positive principal eigenvalue under the constraint that the absolute value of the weight is bounded and the total weight is a fixed negative constant. Biologically, this minimization problem is motivated by the question of determining the optimal spatial arrangement of favorable and unfavorable regions for a species to survive. For rectangular domains with Neumann boundary condition, it is known that there exists a threshold value such that if the total weight is below this threshold value then the optimal favorable region is like a section of a disk at one of the four corners; otherwise, the optimal favorable region is a strip attached to the shorter side of the rectangle. Here, we investigate the same problem with mixed Robin-Neumann type boundary conditions and study how this boundary condition affects the optimal spatial arrangement.

Keywords. Asymptotic analysis, principal eigenvalue, elliptic boundary value problem with indefinite weights, Robin conditions, shape optimization.
\end{abstract}

AMS subject classifications. 35P15, 35J20, 49Q10, 92D25

\section{Introduction: Indefinite Eigenvalue Problem}

The following linear eigenvalue problem with indefinite weight is of particular interest in the study of population dynamics [13]:

$$
\left\{\begin{aligned}
\Delta \varphi+\lambda m \varphi & =0 \text { in } \Omega, \\
\partial_{n} \varphi+\beta \varphi & =0 \text { on } \partial \Omega,
\end{aligned}\right.
$$

where $\Omega$ is a bounded domain in $\mathbb{R}^{N}$ with a smooth boundary $\partial \Omega, n$ is the outward unit normal vector on $\partial \Omega$, and the weight $m$ is a bounded measurable function which changes sign in $\Omega$ and satisfies

$$
-1 \leq m(x) \leq \kappa \quad \forall x \text { in } \Omega,
$$

where $\kappa>0$ is a given constant. It is said that $\lambda$ is a principal eigenvalue of (1) if the corresponding eigenfunction $\varphi \in H^{1}(\Omega)$ is positive. The existence of principal eigenvalues of (1) was discussed in $[2,5]$ for different values of $\beta$. It is well know that the case when $0<\beta<\infty$ is similar to the Dirichlet case. Suppose that

$$
\Omega^{+}=\{x \in \Omega: m(x)>0\}
$$


has positive Lebesgue measure, then problem (1) has a unique positive principal eigenvalue and the corresponding eigenfunction $\varphi$ satisfies

$$
\int_{\Omega} m(x) \varphi(x)^{2} d x>0
$$

In the critical case $\beta=0$, which corresponds to Neumann boundary conditions, 0 is a principal eigenvalue and there is a positive principal eigenvalue if and only if

$$
\int_{\Omega} m(x) d x<0 \quad \text { and } \Omega^{+} \text {has positive Lebesgue measure. }
$$

For $\beta<0$, it was shown in [2] that, depending on $\beta$, (1) has two, one or zero principal eigenvalues. In the case of two principal eigenvalues, the way of distinguishing between them is by considering the sign of $\int_{\Omega} m(x) \varphi(x)^{2} d x$.

One of the motivations for studying the dependence of the principal eigenvalue $\lambda=\lambda(\mathrm{m})$ on the weight $m$ comes from the diffusive logistic equation introduced in [23]:

$$
\begin{cases}u_{t}=\Delta u+\omega u[m(x)-u] & \text { in } \Omega \times \mathbb{R}^{+}, \\ \partial_{n} u+\beta u=0 & \text { on } \partial \Omega \times \mathbb{R}^{+}, \\ u(x, 0) \geq 0, \quad u(x, 0) \not \equiv 0 & \text { in } \bar{\Omega},\end{cases}
$$

where $u(x, t)$ represents the density of a species at location $x$ and time $t$, and $\omega$ is a positive parameter. On one hand, the case $\beta=0$ corresponds to Neumann or no-flux boundary condition and means that the boundary acts as a barrier, i.e. any individual reaching the boundary returns to the interior. On the other hand, the case $\beta=+\infty$ corresponds to Dirichlet conditions and may be interpreted as a deadly boundary, i.e. the exterior environment is completely hostile and any individual reaching the boundary dies. For values $0<\beta<\infty$, we are in the situation where the domain $\Omega$ is surrounded by a partially inhospitable region, where inhospitableness grows with $\beta$. The weight $m$ represents the intrinsic growth rate of species: it is positive in the favorable part of habitat $\left(\Omega_{+}\right)$and negative in the unfavorable one $\left(\Omega_{-}=\{x \in \Omega: m(x)<0\}\right)$. The integral of $m$ over $\Omega$ measures the total resources in a spatially heterogeneous environment.

The logistic equation (4) plays an important role in studying the effects of dispersal and spatial heterogeneity in population dynamics; see, e.g. $[6,7,9]$ and the references therein. It is known that if $\omega \leq \lambda(m)$, then $u(x, t) \rightarrow 0$ uniformly in $\bar{\Omega}$ as $t \rightarrow \infty$ for all nonnegative and non-trivial initial data, i.e., the species goes to extinction; if $\omega>\lambda(m)$, then $u(x, t) \rightarrow u^{*}(x)$ uniformly in $\bar{\Omega}$ as $t \rightarrow \infty$, where $u^{*}$ is the unique positive steady solution of (4) in $W^{2, q}(\Omega)$ for every $q>1$, i.e., the species survives. We are particularly concerned with the effects of spatial variation in the environment of species extinctions. In this connection, let $m_{0}<1$ be a positive constant and assume that

(A1) $m$ satisfies (2), $\Omega^{+}$has positive measure, and $\int_{\Omega} m \leq-m_{0}|\Omega|$.

Since the species can be maintained if and only if $\omega>\lambda(m)$, we see that the smaller $\lambda(m)$ is, the more likely the species can survive. With this in mind, the following question was 
raised and addressed by Cantrell and Cosner in [6, 7]: Among all functions $m$ that satisfy (A1), which $m$ will yield the smallest principal eigenvalue $\lambda(m)$ ? From the biological point of view, finding such a minimizing function $m$ is equivalent to determining the optimal spatial arrangement of the favorable and unfavorable parts of the habitat for species to survive. This issue is important for public policy decisions on conservation of species with limited resources.

Given $m_{0}<1$ and $\kappa>0$, we define the set of admissible functions

$$
\mathcal{M}=\left\{m \in L^{\infty}(\Omega): m \text { satisfies }(\mathrm{A} 1)\right\},
$$

and the problem

$$
\inf \lambda(m) \text { subject to } m \in \mathcal{M} \text {. }
$$

The following result was established in [20] for Neumann conditions (i.e. $\beta=0$ ) and may be straightforwardly extended to Robin conditions, therefore we do not reproduce here the proof but rather refer to [20, Theorem 1.1] for details. This theorem states that the optimal solution $m$ only takes the two barrier values -1 and $\kappa$. Therefore, the analysis may be performed in the set of piecewise constant functions $m$ taking the values -1 and $\kappa$ and verifying the volume constraint instead of $\mathcal{M}$. In this way the problem can be seen as a free boundary problem, where one seeks the interface between the regions where $m$ is equal to -1 or $\kappa$.

Theorem 1. The infimum $\lambda_{\text {inf }}:=\inf _{m \in \mathcal{M}} \lambda(m)$ is attained at some $m \in \mathcal{M}$. Moreover, if $\lambda(m)=\lambda_{\text {inf }}$, then $m$ can be represented as $m=\kappa \chi_{E}-\chi_{\Omega \backslash E}$ almost everywhere (a.e.) in $\Omega$ for some measurable set $E \subset \Omega$. In addition, the volume constraint in (A1) is active, i.e. $\int_{\Omega} m=-m_{0}|\Omega|$.

The fact that the volume constraint in (A1) is active is due to a comparison principle (see [20, Lemma 2.3]) which establishes the property:

$$
m_{1}>m_{2} \Rightarrow \lambda\left(m_{1}\right)<\lambda\left(m_{2}\right) .
$$

This comparison principle is obtained straightforwardly by comparing the Rayleigh quotient (75) for $m_{1}$ and $m_{2}$. In view of this principle, it is clear that the volume constraint must be active at the optimum, otherwise the eigenvalue could still be further decreased.

In this paper we assume $\beta \geq 0$ and use the notation $m_{E}:=\kappa \chi_{E}-\chi_{\Omega \backslash E}$. In section 2 , in one dimension problem (6) with Robin boundary conditions is solved in the case where $E$ has exactly one connected component. We show that there is a threshold value $\beta^{*}$ such that the optimal set behaves like in the case of Dirichlet boundary conditions for $\beta>\beta^{*}$ and like in the case of Neumann boundary conditions for $\beta<\beta^{*}$. From section 3 on, we consider multi-dimensional domains. In particular, cylindrical domain in $\mathbb{R}^{N}$ with mixed Robin-Neumann conditions are analysed. The asymptotic analysis for a small perturbation of $m_{E}$ with $E$ being a strip is performed and the second-order derivative $\lambda_{2}$ of $\lambda$ is derived. The knowledge of the sign of $\lambda_{2}$ allows to determine whether $m_{E}$ is a local optimum for problem (6). In section 4, the sign of $\lambda_{2}$ is analysed using the results of section 2 in one dimension. Conditions proving the strip $E$ to be optimal are analysed in sections 5 and 6 . 
Finally, in section 7 a numerical method for minimizing the eigenvalue is given and applied in the one- and two-dimensional cases, respectively.

\section{Principal eigenvalue with Robin conditions in one Dimension}

In this section we study the one-dimensional case, i.e. $N=1$. Without loss of generality, we take $\Omega=(0,1)$. According to Theorem 1, we consider the minimization of $\lambda(m)$ with $m=\kappa \chi_{E}-\chi_{\Omega \backslash E}, E \in S_{c}$, where

$$
S_{c}:=\{E \subset \Omega, E \text { measurable }:|E|=c\},
$$

and $c$ is such that (A1) is satisfied and active, i.e. $\int_{\Omega} m=-m_{0}|\Omega|$. The case of Robin boundary conditions contains the Dirichlet case, for $\beta=\infty$, and the Neumann case for $\beta=0$. The Neumann case in one dimension was fully investigated in [20], where the authors give the explicit global optimum of the principal eigenvalue. The method consists in looking for minimizers into the subsets $S_{c}^{k}$ of $S_{c}$ given by

$$
S_{c}^{k}:=\left\{E \in S_{c}: E \text { consists of } k \text { disjoint open intervals }\right\} \text { for } k \in \mathbb{N} \text {. }
$$

Unfortunately, this method relies on the extensive use of the Neumann conditions and cannot be transferred to Robin boundary conditions. In the Robin case, we are only able to obtain optimal sets if we minimize $\lambda(m)$ in the smaller set $S_{c}^{1}$ instead of $S_{c}$. Nevertheless, it is conjectured, in view of the Neumann [20] and Dirichlet [11] cases, as well as the numerical results from section 7 , that the optimal sets are indeed in $S_{c}^{1}$ when minimizing in $S_{c}$. Therefore, in what follows, we consider sets $E \in S_{c}^{1}$.

In this section, based on a particular ansatz for the solution of (8)-(9) below, we obtain equation (17) determining the eigenvalue. It cannot be solved explicitly, however studying this equation allows us to determine the monotonicity of $\lambda$ with respect to the position of $E$ and thus to conclude on the optimality of $E$ in $S_{c}^{1}$. These results are given in Theorem 2. In [8, section 2], the one-dimensional version of problem (1) is studied in the special case $\kappa=1$. Here, we study the general case $\kappa>0$. Then the eigenvalue problem (1) becomes

$$
\begin{aligned}
\varphi^{\prime \prime}+\lambda m \varphi & =0 \quad \text { in }(0,1), \\
\varphi^{\prime}(0)-\beta \varphi(0)=\varphi^{\prime}(1)+\beta \varphi(1) & =0 .
\end{aligned}
$$

Let $0<a<b<1$ and $c=b-a>0$. The interval $E$ is defined as $E:=(a, b)$ and $m$ as $m:=\kappa \chi_{E}-\chi_{\Omega \backslash E}, E \in S_{c}^{1}$. We may rewrite the eigenvalue problem (1) as

$$
\begin{aligned}
\varphi^{\prime \prime}-\lambda \varphi & =0 & & \text { in }(0, a), \\
\varphi^{\prime \prime}+\lambda \kappa \varphi & =0 & & \text { in }(a, b), \\
\varphi^{\prime \prime}-\lambda \varphi & =0 & & \text { in }(b, 1), \\
\varphi^{\prime}(0)-\beta \varphi(0)=\varphi^{\prime}(1)+\beta \varphi(1) & =0 & & \\
\varphi^{\prime}(a)^{+}-\varphi^{\prime}(a)^{-}=\varphi^{\prime}(b)^{+}-\varphi^{\prime}(b)^{-} & =0 & & \\
\varphi(a)^{+}-\varphi(a)^{-}=\varphi(b)^{+}-\varphi(b)^{-} & =0 & &
\end{aligned}
$$


According to $[2,5]$ we have $\lambda>0, \varphi>0$ for $\beta \geq 0$ and we may consider

$$
\varphi(x)=\left\{\begin{array}{ccc}
C_{1} \cosh \sqrt{\lambda}(x-a)+C_{2} \sinh \sqrt{\lambda}(x-a) & \text { in } \quad(0, a), \\
C_{3} \cos \sqrt{\lambda \kappa}(x-a)+C_{4} \sin \sqrt{\lambda \kappa}(x-a) & \text { in }(a, b), \\
C_{5} \cosh \sqrt{\lambda}(x-b)+C_{6} \sinh \sqrt{\lambda}(x-b) & \text { in } \quad(b, 1),
\end{array}\right.
$$

with constants $C_{i}=C_{i}(E), i=1, . ., 6$. The boundary and transmission conditions (13)-(15) may be written as a system $M C=0$, where $M$ is a $6 \times 6$ matrix with coefficients $m_{i j}$ and $C$ is a column vector whose elements are $C_{i}, i=1, . .6$. The coefficients of $M$ are all zeros except for

$$
\begin{array}{llll}
m_{11} & =\frac{\sqrt{\lambda} \sinh (\sqrt{\lambda} a)+\beta \cosh (\sqrt{\lambda} a)}{\sqrt{\lambda} \cosh (\sqrt{\lambda} a)+\beta \sinh (\sqrt{\lambda} a)}, & & m_{12}=-1, \\
m_{25} & =\frac{\sqrt{\lambda} \sinh (\sqrt{\lambda}(1-b))+\beta \cosh (\sqrt{\lambda}(1-b))}{\sqrt{\lambda} \cosh (\sqrt{\lambda}(1-b))+\beta \sinh (\sqrt{\lambda}(1-b))}, & & m_{26}=1, \\
m_{31} & =1, & & m_{33}=-1, \\
m_{42} & =-1, & m_{44}=\sqrt{\kappa}, & \\
m_{53}=\cos \sqrt{\lambda \kappa} c, & m_{54}=\sin \sqrt{\lambda \kappa} c, & m_{55}=-1, \\
m_{63}=-\sqrt{\kappa} \sin \sqrt{\lambda \kappa} c, & m_{64}=\sqrt{\kappa} \cos \sqrt{\lambda \kappa} c, \quad m_{66}=-1 .
\end{array}
$$

Obviously, $M C=0$ has a non-trivial solution only if $\operatorname{det} M=0$. If this is the case, then one may give an expression of the eigenfunction $\varphi$ proportional to $C_{1} \neq 0$. The coefficient $C_{1}$ is then uniquely defined by a normalization condition on $\varphi$, for instance $\int_{\Omega} \varphi=1$. Actually, solving the equation $\operatorname{det} M=0$ yields the eigenvalue $\lambda$. With some elementary but laborious calculations, this equation becomes

$$
f(a, \beta, \kappa, \lambda)=0
$$

with $f$ given by

$$
\begin{aligned}
f(a, \beta, \kappa, \lambda)=\quad \cosh (\sqrt{\lambda}(1-a-c)) \cdot\left[\cosh (\sqrt{\lambda} a)\left(l_{s}\left(-\lambda \sqrt{\kappa}+\beta^{2} / \sqrt{\kappa}\right)+l_{c}(2 \beta \sqrt{\lambda})\right)\right. \\
\left.+\sinh (\sqrt{\lambda} a)\left(l_{s}(-\beta \sqrt{\lambda \kappa}+\beta \sqrt{\lambda} / \sqrt{\kappa})+l_{c}\left(\lambda+\beta^{2}\right)\right)\right] \\
+\sinh (\sqrt{\lambda}(1-a-c)) \cdot\left[\cosh (\sqrt{\lambda} a)\left(l_{s}(-\beta \sqrt{\lambda \kappa}+\beta \sqrt{\lambda} / \sqrt{\kappa})+l_{c}\left(\lambda+\beta^{2}\right)\right)\right. \\
\left.+\sinh (\sqrt{\lambda} a)\left(l_{s}\left(-\beta^{2} \sqrt{\kappa}+\lambda / \sqrt{\kappa}\right)+l_{c}(2 \beta \sqrt{\lambda})\right)\right]
\end{aligned}
$$

where we have used the notations $l_{c}:=\cos (\sqrt{\lambda \kappa} c)$ and $l_{s}:=\sin (\sqrt{\lambda \kappa} c)$. Theorem 1 and (A1) imply that $c$ depends on $\kappa$ in the following way:

$$
c=\frac{1-m_{0}}{1+\kappa}<\frac{1}{1+\kappa} .
$$


When $\beta=0$, then (17) becomes the characteristic equation for the Neumann problem

$$
\tanh \sqrt{\lambda}(1-c)=\frac{\sqrt{\kappa} \tan (\sqrt{\lambda \kappa} c)-\tanh (\sqrt{\lambda} a)}{1+\tanh (\sqrt{\lambda} a) \tan (\sqrt{\lambda \kappa} c) / \sqrt{\kappa}} .
$$

which, for $a=0$, leads to the formula that was found in [20], i.e.

$$
\sqrt{\kappa} \tan \sqrt{\lambda \kappa} c=\tanh \sqrt{\lambda}(1-c) \text {. }
$$

When $\beta \rightarrow \infty$, (17) also provides a characteristic equation for the Dirichlet problem:

$$
\tanh \sqrt{\lambda}(1-c)=-\frac{\tan (\sqrt{\lambda \kappa} c) / \sqrt{\kappa}+\tanh (\sqrt{\lambda} a)}{1-\sqrt{\kappa} \tanh (\sqrt{\lambda} a) \tan (\sqrt{\lambda \kappa} c)}
$$

which, for $a=0$, leads to the relation

$$
\tan \sqrt{\lambda \kappa} c=-\sqrt{\kappa} \tanh \sqrt{\lambda}(1-c) .
$$

Remark 1. Equation (19) has a unique solution $\lambda$ in $] 0,(\pi / 2 c)^{2} / \kappa[$, while (21) has a unique solution in $](\pi / 2 c)^{2} / \kappa,(\pi / c)^{2} / \kappa[$. According to $[1], \lambda$ is an increasing function of $\beta$. This indicates that $0<\lambda<(\pi / c)^{2} / \kappa$ for any $\beta$.

Notice that $f$ can also be expressed in the following way:

$$
f(a, \beta, \kappa, \lambda)=g(\beta, \kappa, \lambda)+h(a, \beta, \kappa, \lambda),
$$

with

$$
\begin{aligned}
g(\beta, \kappa, \lambda)= & 2 \beta \sqrt{\lambda} l_{c} \cosh (\sqrt{\lambda}(1-c))+\beta \sqrt{\lambda}(1 / \sqrt{\kappa}-\sqrt{\kappa}) l_{s} \sinh (\sqrt{\lambda}(1-c)) \\
& +\left(\lambda+\beta^{2}\right) l_{c} \sinh (\sqrt{\lambda}(1-c))+\lambda(1 / \sqrt{\kappa}-\sqrt{\kappa}) l_{s} \cosh (\sqrt{\lambda}(1-c)), \\
h(a, \beta, \kappa, \lambda)= & \cosh (\sqrt{\lambda}(1-a-c)) \cosh (\sqrt{\lambda} a) l_{s}\left(\beta^{2}-\lambda\right) / \sqrt{\kappa} \\
& +\sinh (\sqrt{\lambda}(1-a-c)) \sinh (\sqrt{\lambda} a) l_{s}\left(\lambda-\beta^{2}\right) \sqrt{\kappa} .
\end{aligned}
$$

After simplification, the partial derivative of $f$ with respect to $a$ is given by

$$
\partial_{a} f(a, \beta, \kappa, \lambda)=\left(\beta^{2}-\lambda\right)\left(\sqrt{\lambda} l_{s}(\sqrt{\kappa}+1 / \sqrt{\kappa})\right)(\sinh \sqrt{\lambda}(a-(1-a-c))) .
$$

According to Remark 1 we have $0<\lambda<(\pi / c)^{2} / \kappa$, thus in (23) the sign of $\partial_{a} f(a, \beta, \kappa, \lambda)$ depends only on the sign of $\beta^{2}-\lambda$ and $a-(1-c) / 2$. It is known [16] that for $\beta>0,(8)-(9)$ has a unique positive principal eigenvalue, which is also the first positive eigenvalue of (8)(9). Therefore, for each $0 \leq a \leq 1-c, \beta \geq 0, \kappa>0$ there exists $\lambda(a, \beta, \kappa)$ such that (17) is satisfied with the associated eigenfunction $\varphi$ fulfilling $\varphi>0$. Considering $\lambda=\lambda(a, \beta, \kappa)$ and taking the derivative with respect to $a$ we obtain

$$
\partial_{\lambda} f(a, \beta, \kappa, \lambda(a, \beta, \kappa)) \partial_{a} \lambda(a, \beta, \kappa)=-\partial_{a} f(a, \beta, \kappa, \lambda(a, \beta, \kappa)) .
$$

Thus, in order to determine the sign of $\partial_{a} \lambda(a, \beta, \kappa)$ we need to establish the sign of $\partial_{\lambda} f(a, \beta, \kappa, \lambda(a, \beta, \kappa))$. However, the derivative with respect to $\lambda$ of $f$ is very involved as can be seen from the definition of $f$. Instead, to determine $\partial_{\lambda} f$ we may write the derivative with respect to $\beta$ of $f$ and we get in a similar way:

$$
\partial_{\lambda} f(a, \beta, \kappa, \lambda(a, \beta, \kappa)) \partial_{\beta} \lambda(a, \beta, \kappa)=-\partial_{\beta} f(a, \beta, \kappa, \lambda(a, \beta, \kappa)) .
$$


It has been shown in [1] that $\partial_{\beta} \lambda(a, \beta, \kappa)>0$, thus $\partial_{\lambda} f(a, \beta, \kappa, \lambda(a, \beta, \kappa))$ and $\partial_{\beta} f(a, \beta, \kappa, \lambda(a, \beta, \kappa))$ have opposite signs. Now we compute the derivative of $f$ with respect to $\beta$. We obtain, using (22)

$$
\begin{aligned}
\partial_{\beta} f(a, \beta, \kappa, \lambda)= & 2 \sqrt{\lambda} l_{c} \cosh (\sqrt{\lambda}(1-c))+\sqrt{\lambda}(1 / \sqrt{\kappa}-\sqrt{\kappa}) l_{s} \sinh (\sqrt{\lambda}(1-c)) \\
& +2 \beta l_{c} \sinh (\sqrt{\lambda}(1-c)) \\
& +2 \cosh (\sqrt{\lambda}(1-a-c)) \cosh (\sqrt{\lambda} a) l_{s} \beta / \sqrt{\kappa} \\
& -2 \sinh (\sqrt{\lambda}(1-a-c)) \sinh (\sqrt{\lambda} a) l_{s} \beta \sqrt{\kappa}
\end{aligned}
$$

We study the case $\beta=\sqrt{\lambda}$. In view of $(23)$ and (24) we have

$$
\partial_{a} \lambda(a, \beta, \kappa)=\partial_{a} f(a, \beta, \kappa, \lambda(a, \beta, \kappa))=0,
$$

and $\lambda(a, \beta, \kappa)$ is consequently constant with respect to $a$, which means that any domain $E$ in the class $S_{c}^{1}$ is optimal if there exists a $\beta^{*}$ such that $\sqrt{\lambda\left(a, \beta^{*}, \kappa\right)}=\beta^{*}$. In view of $(22)$ we have that

$$
\begin{aligned}
f\left(a, \beta, \kappa, \beta^{2}\right)= & 2 \beta^{2} \cos (c \beta \sqrt{\kappa})(\cosh (\beta(1-c))+\sinh (\beta(1-c))) \\
& +\beta^{2}(1 / \sqrt{\kappa}-\sqrt{\kappa}) \sin (c \beta \sqrt{\kappa})(\cosh (\beta(1-c))+\sinh (\beta(1-c))) \\
= & e^{\beta(1-c)}\left(2 \beta^{2} \cos (c \beta \sqrt{\kappa})+\beta^{2}(1 / \sqrt{\kappa}-\sqrt{\kappa}) \sin (c \beta \sqrt{\kappa})\right) .
\end{aligned}
$$

If $\kappa=1$, the smallest solution to $f\left(a, \beta, \kappa, \beta^{2}\right)=0$ is

$$
\beta=\beta^{*}=\pi /(2 c \sqrt{\kappa})=\pi /(2 c) .
$$

If $\kappa>1$, the smallest solution of $f\left(a, \beta, \kappa, \beta^{2}\right)=0$ is given by

$$
\beta=\beta^{*}=\frac{1}{c \sqrt{\kappa}} \arctan \frac{2 \sqrt{\kappa}}{\kappa-1}=\frac{2}{c \sqrt{\kappa}} \arctan \frac{1}{\sqrt{\kappa}} .
$$

Finally, if $\kappa<1$, the smallest solution of $f\left(a, \beta, \kappa, \beta^{2}\right)=0$ is given by

$$
\beta=\beta^{*}=\frac{1}{c \sqrt{\kappa}}\left(\arctan \frac{2 \sqrt{\kappa}}{\kappa-1}+\pi\right) .
$$

We may now state the main result of this section.

Theorem 2. The principal eigenvalue $\lambda(a, \beta, \kappa)$ is continuous with respect to $\beta$ for $\beta \geq 0$. It is also symmetric with respect to $a=(1-c) / 2$ for $0 \leq a \leq 1-c$ and:

- if $\beta>\beta^{*}$, then $\lambda(a, \beta, \kappa)$ is strictly decreasing with respect to a, i.e. $\partial_{a} \lambda(a, \beta, \kappa)<0$, for $0 \leq a \leq(1-c) / 2$; therefore the minimum of $\lambda(a, \beta, \kappa)$ is attained for $a=$ $(1-c) / 2$.

- if $\beta<\beta^{*}$, then $\lambda(a, \beta, \kappa)$ is strictly increasing with respect to a, i.e. $\partial_{a} \lambda(a, \beta, \kappa)>0$, for $0 \leq a \leq(1-c) / 2$; therefore the minimum of $\lambda(a, \beta, \kappa)$ is attained for $a=0$ and $a=1-c$.

- if $\beta=\beta^{*}$, then $\lambda(a, \beta, \kappa)$ is constant with respect to $a$ and any $0 \leq a \leq 1-c$ is a global minimum for $\lambda(a, \beta, \kappa)$. 
Proof. We start with the continuity. The symmetry of $\lambda(a, \beta, \kappa)$ with respect to $a=$ $(1-c) / 2$ is obvious. Therefore we may assume that $0 \leq a<(1-c) / 2$. For fixed $a$ and $\kappa$, we define the function $\hat{f}: \mathbb{R}_{+}^{2} \ni(\beta, \lambda) \mapsto f(a, \beta, \kappa, \lambda) \in \mathbb{R}$. In view of the definition of $f, \hat{f}$ is clearly a $\mathcal{C}^{\infty}$-function on $\mathbb{R}_{+}^{2}$. Due to $(23)-(24)$ and $a<(1-c) / 2$ we have

$$
\partial_{\lambda} \hat{f}(\beta, \lambda)=0 \Rightarrow \beta=\beta^{*}=\sqrt{\lambda\left(a, \beta^{*}, \kappa\right)} .
$$

Therefore, we may apply the implicit function theorem on $] 0, \beta^{*}[$ and $] \beta^{*},+\infty[$ to obtain the existence of a function $\lambda(\beta)$ of class $\mathcal{C}^{\infty}$ on $] 0, \beta^{*}[$ and $] \beta^{*},+\infty[$ such that

$$
\hat{f}(\beta, \lambda(\beta))=0 \text { on } \mathbb{R}^{+} \backslash\left\{\beta^{*}\right\} \text {. }
$$

For $0<\kappa \leq 1$, one easily finds that $\partial_{\lambda} \hat{f}(\beta, \lambda)>0$ for all $\beta \in \mathbb{R}_{+}$. Therefore we have $\lambda(\beta) \in \mathcal{C}^{\infty}\left(\mathbb{R}_{+}, \mathbb{R}\right)$ for $0<\kappa \leq 1$. For $\kappa>1$, the continuity on $\mathbb{R}_{+}$will be obtained below.

We now show the monotonicity of the eigenvalue with respect to $\beta$. First of all, in view of $(24),(25)$, and since $\partial_{\beta} \lambda(a, \beta, \kappa)>0$ as proved in [1], we have the following sequence of implications

$$
\partial_{\beta} f(a, \beta, \kappa, \lambda(a, \beta, \kappa))=0 \Rightarrow \partial_{\lambda} f(a, \beta, \kappa, \lambda(a, \beta, \kappa))=0 \Rightarrow \partial_{a} f(a, \beta, \kappa, \lambda(a, \beta, \kappa))=0 .
$$

According to $(23)$ and due to $0<\lambda<(\pi / c)^{2} / \kappa$ we have

$$
\partial_{a} f(a, \beta, \kappa, \lambda(a, \beta, \kappa))=0 \text { and } 0 \leq a \leq(1-c) / 2 \Leftrightarrow \beta=\sqrt{\lambda(a, \beta, \kappa)} .
$$

We have seen above that $\beta=\sqrt{\lambda}$ is equivalent to $\beta=\beta^{*}$. Thus,

$$
\partial_{\beta} f(a, \beta, \kappa, \lambda(a, \beta, \kappa))=0 \Rightarrow \beta=\beta^{*}=\sqrt{\lambda\left(a, \beta^{*}, \kappa\right)} .
$$

Since $\partial_{\beta} f(a, \beta, \kappa, \lambda(a, \beta, \kappa))$ is linear with respect to $\beta$, it does not change sign on the halfline $\beta>\beta^{*}$ and on the segment $0<\beta<\beta^{*}$. First assume that $\beta>\beta^{*}$. This implies that $\beta>\sqrt{\lambda(a, \beta, \kappa)}$. Indeed, assume that $\beta<\sqrt{\lambda(a, \beta, \kappa)}$; the case $\beta=\sqrt{\lambda(a, \beta, \kappa)}$ is obviously ruled out because it implies $\beta=\beta^{*}$. Since $\lambda(a, \beta, \kappa)$ is strictly monotone with respect to $\beta, \lambda(a, \beta, \kappa)$ is bounded with respect to the principal eigenvalue of the Dirichlet problem, i.e. problem (8)-(9) with $\beta=\infty$. Thus, the principal eigenvalue $\lambda$ is bounded as a function of $\beta$. Now, if there exists $\beta^{*}<\beta<\sqrt{\lambda(a, \beta, \kappa)}$, then invoking the continuity of $\lambda(a, \beta, \kappa)$ with respect to $\beta$ on $] \beta^{*},+\infty\left[\right.$, there exists $\beta_{0}$ such that $\beta^{*}<\beta_{0}=\sqrt{\lambda\left(a, \beta_{0}, \kappa\right)}$. However we have seen that in this case we necessarily have $\beta_{0}=\beta^{*}$ which contradicts $\beta^{*}<\beta_{0}$.

According to $(25), \partial_{\beta} f(a, \beta, \kappa, \lambda(a, \beta, \kappa))$ and $\partial_{\lambda} f(a, \beta, \kappa, \lambda(a, \beta, \kappa))$ have opposite signs. In view of (23) and (24) and thanks to $\beta>\sqrt{\lambda(a, \beta, \kappa)}$ we then have two cases:

(1) if $\partial_{\beta} f(a, \beta, \kappa, \lambda(a, \beta, \kappa))>0$, then $\partial_{a} \lambda(a, \beta, \kappa)<0$,

(2) if $\partial_{\beta} f(a, \beta, \kappa, \lambda(a, \beta, \kappa))<0$, then $\partial_{a} \lambda(a, \beta, \kappa)>0$.

The second case is not possible since for $\beta=+\infty$, which corresponds to Dirichlet boundary conditions, it is known [19] that we should have $\partial_{a} \lambda(a, \beta, \kappa)<0$. Therefore we necessarily have $\partial_{a} \lambda(a, \beta, \kappa)<0$ if $\beta>\beta^{*}$.

Now we look at the case $\beta<\beta *$. This implies that $\beta<\sqrt{\lambda(a, \beta, \kappa)}$. Indeed, assume that $\beta>\sqrt{\lambda(a, \beta, \kappa)}$ (the case $\beta=\sqrt{\lambda(a, \beta, \kappa)}$ is obviously ruled out). The principal eigenvalue 
$\lambda$ has a positive lower bound as a function of $\beta$; see [22] for details. Thus, if there exists $\beta^{*}>\beta>\sqrt{\lambda(a, \beta, \kappa)}$, then invoking the continuity of $\lambda(a, \beta, \kappa)$ with respect to $\beta$ on $] 0, \beta^{*}[$, there exists $\beta_{0}$ such that $\beta^{*}>\beta_{0}=\sqrt{\lambda\left(a, \beta_{0}, \kappa\right)}$. However we have seen that in this case we necessarily have $\beta_{0}=\beta^{*}$ which contradicts $\beta^{*}>\beta_{0}$.

In view of $(23),(24),(25)$ and thanks to $\beta<\sqrt{\lambda(a, \beta, \kappa)}$ we have two cases:

(1) if $\partial_{\beta} f(a, \beta, \kappa, \lambda(a, \beta, \kappa))>0$ then $\partial_{a} \lambda(a, \beta, \kappa)>0$,

(2) if $\partial_{\beta} f(a, \beta, \kappa, \lambda(a, \beta, \kappa))<0$ then $\partial_{a} \lambda(a, \beta, \kappa)<0$.

The second case is not possible since it includes the case $\beta=0$ (Neumann boundary conditions), and it is known [20] that we should have $\partial_{a} \lambda(a, \beta, \kappa)>0$ in this case. Therefore we necessarily have $\partial_{a} \lambda(a, \beta, \kappa)>0$ if $\beta<\beta^{*}$.

Finally, since $\beta<\sqrt{\lambda(a, \beta, \kappa)}$ on $] 0, \beta^{*}[$ and $\beta>\sqrt{\lambda(a, \beta, \kappa)}$ on $] \beta^{*}, \infty[$, the monotonicity of $\lambda(a, \beta, \kappa)$ with respect to $\beta$ implies that $\lambda(a, \beta, \kappa)$ is continuous with respect to $\beta$ also at $\beta=\beta^{*}$.

\section{Cylindrical domains}

In section 2 we have obtained pivotal results about the optimal weight function $m$ in one dimension. Using this knowledge, we would like to move on to multi-dimensional problems. Unlike in one dimension, it is not possible to perform explicit calculations, unless we consider particular geometries for $\Omega$. Thus, as a first step towards the study of general multi-dimensional domains, we propose to look at cylindrical domains in $\mathbb{R}^{N}$ with mixed Robin-Neumann conditions. Indeed, this allows to separate variables and exploit the results in one dimension obtained in section 2. On the top and bottom of the cylinder we impose Robin boundary conditions while on the lateral boundary Neumann conditions are prescribed. The problem with Neumann boundary conditions on the whole boundary was studied in [17].

We determine if the "strip" touching the shorter side of the rectangle is a local optimizer or not for the minimization problem (6). For this purpose, in this section we introduce the strip $\Omega_{0}^{+}$corresponding to $m=\kappa$ and consider small perturbations $\Omega_{\varepsilon}^{+}$of $\Omega_{0}^{+}$in terms of a function $g$ and a small factor $\varepsilon$. Using classical methods of asymptotic analysis, we obtain asymptotic expansions of $\lambda_{\varepsilon}$ and $\varphi_{\varepsilon}$, respectively the eigenvalue and eigenvector corresponding to $\Omega_{\varepsilon}^{+}$, with respect to $\varepsilon$. The subsequent sections are dedicated to determining the optimality of this strip using the obtained asymptotic expansions.

Let $\Omega$ be a cylindrical domain in $\mathbb{R}^{N}$ given by

$$
\Omega:=(0,1) \times D \subset \mathbb{R}^{N}, \quad N \geq 2,
$$

where $D$ is a bounded domain in $\mathbb{R}^{N-1}$ with smooth boundary $\partial D$. We denote by $\Sigma:=$ $\{0,1\} \times D$ and $\Gamma:=(0,1) \times \partial D$ the top and bottom and the lateral boundary of the cylinder $\Omega$, respectively. Let $\Omega_{0}^{+}$and $\Omega_{0}^{-}$be subsets of $\Omega$ defined by

$$
\Omega_{0}^{+}:=(0, c) \times D, \quad \Omega_{0}^{-}:=(c, 1) \times D
$$


with a parameter $c \in(0,1)$, and set $m(x, y)=\kappa$ if $(x, y) \in \Omega_{0}^{+}$and $m(x, y)=-1$ if $(x, y) \in$ $\Omega_{0}^{-}$, where $x \in(0,1)$ and $y \in D$. Note that $(3)$ is equivalent to $0<c<c^{*}:=(\kappa+1)^{-1}$.

We perturb the problem (1) as follows. Let $g: D \rightarrow \mathbb{R}$ be any $L^{2}$-function satisfying

$$
\int_{D} g(y) d y=0
$$

and define the perturbed domain $\Omega=\Omega_{\varepsilon}^{+} \cup \Omega_{\varepsilon}^{-}$with

$$
\begin{aligned}
& \Omega_{\varepsilon}^{+}:=\left\{(x, y) \in \mathbb{R} \times \mathbb{R}^{N-1}: 0<x<c+\varepsilon g(y), y \in D\right\}, \\
& \Omega_{\varepsilon}^{-}:=\left\{(x, y) \in \mathbb{R} \times \mathbb{R}^{N-1}: c+\varepsilon g(y)<x<1, y \in D\right\},
\end{aligned}
$$

where $\varepsilon>0$ is a small parameter. Then set $m_{\varepsilon}(x, y)=\kappa$ if $(x, y) \in \Omega_{\varepsilon}^{+}, m_{\varepsilon}(x, y)=-1$ if $(x, y) \in \Omega_{\varepsilon}^{-}$and consider the perturbed problem

$$
\begin{cases}\Delta \varphi_{\varepsilon}+\lambda_{\varepsilon} m_{\varepsilon} \varphi_{\varepsilon}=0 & \text { in } \Omega, \\ \partial_{n} \varphi_{\varepsilon}+\beta \varphi_{\varepsilon}=0 & \text { on } \Sigma, \\ \partial_{n} \varphi_{\varepsilon}=0 & \text { on } \Gamma .\end{cases}
$$

For $\varepsilon=0$, the solution $\varphi_{0}$ of $(35)$ is independent of $y$, i.e. $\varphi_{0}(x, y)=: \varphi(x)$. The main goal of this section is to find a formal asymptotic expansion of $\lambda_{\epsilon}$ for small $\epsilon>0$. To this end, we consider the following ansätze for $\lambda_{\varepsilon}$ and $\varphi_{\varepsilon}$

$$
\begin{aligned}
& \lambda_{\varepsilon}=\lambda+\varepsilon \lambda_{1}+\varepsilon^{2} \lambda_{2}+\ldots, \\
& \varphi_{\varepsilon}(x, y)=\varphi(x)+\varepsilon \varphi_{1}(x, y)+\varepsilon^{2} \varphi_{2}(x, y)+\ldots,
\end{aligned}
$$

where $(\varphi, \lambda)$ is an eigenpair of the one-dimensional problem (8)-(9). We substitute ansätze (36) into the weak form

$$
-\int_{\Omega} \nabla \varphi_{\varepsilon} \cdot \nabla \psi-\int_{\partial \Omega} \partial_{n} \varphi_{\varepsilon} \psi+\lambda_{\varepsilon} \int_{\Omega} m_{\varepsilon} \varphi_{\varepsilon} \psi=0 \quad \text { for any } \psi \in C^{1}(\bar{\Omega}),
$$

and gather $\varepsilon^{0}-, \varepsilon^{1}$ - and $\varepsilon^{2}$-order terms according to the procedure in [17]. In this way we obtain the equations fulfilled by $\varphi_{1}, \lambda_{1}$ and $\lambda_{2}$. Since the calculations to obtain these equations are similar to those in [17], we do not reproduce them here for the sake of compactness and refer to [17, section 3] for details. We actually obtain $\lambda_{1}=0$ and $\varphi_{1}$ must satisfy the equation

$$
\left\{\begin{aligned}
\Delta \varphi_{1}+\lambda \kappa \varphi_{1} & =0 \text { in }(0, c) \times D \\
\Delta \varphi_{1}-\lambda \varphi_{1} & =0 \text { in }(c, 1) \times D
\end{aligned}\right.
$$

with the boundary conditions

$$
\partial_{n} \varphi_{1}+\beta \varphi_{1}=0 \quad \text { on } \Sigma, \quad \partial_{n} \varphi_{1}=0 \quad \text { on } \Gamma,
$$

and the transmission condition

$$
\frac{\partial \varphi_{1}}{\partial x}\left(\lim _{x \uparrow c} x\right)-\frac{\partial \varphi_{1}}{\partial x}\left(\lim _{x \downarrow c} x\right):=\left.\frac{\partial \varphi_{1}}{\partial x}\right|_{x=c-} ^{x=c+}=-\lambda(\kappa+1) \varphi(c) g(y), \quad y \in D .
$$


Finally, from $\varepsilon^{2}$-order terms, we derive (see [17, section 3]) that $\lambda_{2}$ is expressed as

$$
\lambda_{2}=-\frac{\lambda(\kappa+1) \varphi(c) \int_{D} \varphi_{1}(c, y) g(y)+\varphi^{\prime}(c) g^{2}(y) d y}{\int_{\Omega} m \varphi^{2}} .
$$

Since $\lambda_{1}=0$ for this geometric configuration, $m$ is a candidate for providing a local minimizer of the principal eigenvalue. To determine whether $\lambda$ is indeed a local minimizer we need to study the sign of $\lambda_{2}$. In the following sections we show that the principal eigenvalue $\lambda$ is not locally minimal if

$$
I[g]:=\int_{D} \varphi_{1}(c, y) g(y)+\varphi^{\prime}(c) g^{2}(y) d y>0
$$

for some $g \in L^{2}(D)$.

\section{Determination of the sign of $\lambda_{2}$}

The main result of this section is Lemma 1, where the sign of $\lambda_{2}$ is established depending on the value of $\beta$ and on the smallest positive eigenvalue $\mu$ of the lower-dimensional problem

$$
\left\{\begin{array}{rll}
\Delta_{y} V+\mu V= & \text { in } D \\
\partial_{n} V=0 & \text { on } \partial D .
\end{array}\right.
$$

For the analysis, we compute $\lambda_{2}$ in the particular case where $g(y)$ is an eigenfunction of (42) associated with a positive eigenvalue $\mu>0$. Notice that (34) constitutes a normalization of the eigenfunction in this case. Later on, any function $g$ in $L^{2}$ may be expanded in terms of of eigenfunctions of (42). This enables us to use the results of this section, also in the general case. We set $\varphi_{1}(x, y)=P(x) g(y)$ and infer from (38), (39) and (40) that $P$ satisfies

$$
\left\{\begin{array}{l}
P^{\prime \prime}(x)+(\lambda \kappa-\mu) P(x)=0, \quad 0<x<c, \\
P^{\prime \prime}(x)-(\lambda+\mu) P(x)=0, \quad c<x<1, \\
P^{\prime}(0)-\beta P(0)=P^{\prime}(1)+\beta P(1)=0 \\
P(c+)=P(c-), \\
P^{\prime}(c+)-P^{\prime}(c-)=-\lambda(\kappa+1) \varphi(c) .
\end{array}\right.
$$

Due to the unknown sign of $\lambda \kappa-\mu$ in (43), we compute the solution to (43) by distinguishing the three cases $\mu<\lambda \kappa, \mu=\lambda \kappa$ and $\mu \geq \lambda \kappa$.

4.1. [Case I: $\mu<\lambda \kappa$ ], Computation of $P$. We write $P$ as

$$
P(x)= \begin{cases}C_{3} \cos \sqrt{\lambda \kappa-\mu} x+C_{4} \sin \sqrt{\lambda \kappa-\mu} x, & 0<x<c, \\ C_{5} \cosh \sqrt{\lambda+\mu}(x-1)+C_{6} \sinh \sqrt{\lambda+\mu}(x-1), & c<x<1 .\end{cases}
$$

with constants $C_{i}, i \in\{3,4,5,6\}$. The Robin boundary conditions for $P$ yield

$$
\beta C_{3}=\sqrt{\lambda \kappa-\mu} C_{4}, \quad \beta C_{5}=-\sqrt{\lambda+\mu} C_{6} .
$$


From the transmission condition (40) at $x=c$, we obtain

$$
\begin{aligned}
& C_{5} \sqrt{\lambda+\mu} \sinh (\sqrt{\lambda+\mu}(c-1))+C_{6} \sqrt{\lambda+\mu} \cosh (\sqrt{\lambda+\mu}(c-1)) \\
+ & C_{3} \sqrt{\lambda \kappa-\mu} \sin (\sqrt{\lambda \kappa-\mu} c)-C_{4} \sqrt{\lambda \kappa-\mu} \cos (\sqrt{\lambda \kappa-\mu} c)=-\lambda(\kappa+1) \varphi(c), \\
& C_{3} \cos (\sqrt{\lambda \kappa-\mu} c)+C_{4} \sin (\sqrt{\lambda \kappa-\mu} c) \\
= & C_{5} \cosh (\sqrt{\lambda+\mu}(c-1))+C_{6} \sinh (\sqrt{\lambda+\mu}(c-1)) .
\end{aligned}
$$

For simplicity, we introduce the following notation:

$$
\begin{array}{ll}
h_{c}:=\cosh (\sqrt{\lambda+\mu}(1-c)), & h_{s}:=\sinh (\sqrt{\lambda+\mu}(1-c)), \\
k_{c}:=\cos (\sqrt{\lambda \kappa-\mu} c), & k_{s}:=\sin (\sqrt{\lambda \kappa-\mu} c) .
\end{array}
$$

Observe that $h_{s}=-\sinh (\sqrt{\lambda+\mu}(c-1))$. Using (45)-(47) results in

$$
C_{6}=\frac{\lambda(\kappa+1) \varphi(c) \beta\left(\sqrt{\lambda \kappa-\mu} k_{c}+\beta k_{s}\right)}{\Phi(\mu)} .
$$

with

$$
\begin{aligned}
\Phi(\mu)= & -\left(\sqrt{\lambda \kappa-\mu} k_{c}+\beta k_{s}\right)\left((\lambda+\mu) h_{s}+\beta \sqrt{\lambda+\mu} h_{c}\right) \\
& +\left((\lambda \kappa-\mu) k_{s}-\beta \sqrt{\lambda \kappa-\mu} k_{c}\right)\left(\sqrt{\lambda+\mu} h_{c}+\beta h_{s}\right) .
\end{aligned}
$$

Next, we show that $\Phi(\mu)<0$ for $\lambda \kappa>\mu>0$. With $a=0$, (17) represents the characteristic equation which determines $\lambda(0, \beta, \kappa)$. We actually observe that $\Phi(0)=f(0, \beta, \kappa, \lambda)$ and thus $\Phi(0)=0$, indeed

$$
\Phi(0)=h_{c}^{0}\left[k_{s}^{0}\left(-\lambda \kappa+\beta^{2}\right)+2 \beta \sqrt{\lambda \kappa} k_{c}^{0}\right]+h_{s}^{0}\left[k_{s}^{0}(-\beta \sqrt{\lambda} \kappa+\beta \sqrt{\lambda})+\sqrt{\kappa}\left(\lambda+\beta^{2}\right) k_{c}^{0}\right],
$$

where the notation $h_{c}^{0}, h_{s}^{0}, k_{c}^{0}, k_{s}^{0}$ stands for $h_{c}, h_{s}, k_{s}, k_{s}$ with $\mu=0$. Denoting for $\mu>0$

$$
\Phi_{1}(\mu)=\frac{\Phi(\mu)}{\left(\sqrt{\lambda \kappa-\mu} k_{c}+\beta k_{s}\right)\left(\sqrt{\lambda+\mu} h_{c}+\beta h_{s}\right)}
$$

we get

$$
\begin{aligned}
\Phi_{1}(\mu) & =-\frac{(\lambda+\mu) h_{s}+\beta \sqrt{\lambda+\mu} h_{c}}{\left(\sqrt{\lambda+\mu} h_{c}+\beta h_{s}\right)}+\frac{(\lambda \kappa-\mu) k_{s}-\beta \sqrt{\lambda \kappa-\mu} k_{c}}{\left(\sqrt{\lambda \kappa-\mu} k_{c}+\beta k_{s}\right)} \\
& =-\frac{\sqrt{\lambda+\mu} \tanh \sqrt{\lambda+\mu}(1-c)+\beta}{1+\frac{\beta}{\sqrt{\lambda+\mu}} \tanh \sqrt{\lambda+\mu}(1-c)}+\frac{\sqrt{\lambda \kappa-\mu} \tan \sqrt{\lambda \kappa-\mu} c-\beta}{1+\frac{\beta}{\sqrt{\lambda \kappa-\mu}} \tan \sqrt{\lambda \kappa-\mu} c} .
\end{aligned}
$$

First observe that $\sqrt{\lambda+\mu} \tanh \sqrt{\lambda+\mu}(1-c)$ and $\sqrt{\lambda \kappa-\mu} \tanh \sqrt{\lambda \kappa-\mu} c-\beta$ are strictly increasing and decreasing functions of $\mu$, respectively. Secondly observe that $\frac{\beta}{\sqrt{\lambda+\mu}} \tanh \sqrt{\lambda+\mu}(1-$ $c$ ) is a strictly decreasing function of $\mu$ due to the properties of the tanh-function, whereas $\frac{\beta}{\sqrt{\lambda \kappa-\mu}} \tan \sqrt{\lambda \kappa-\mu} c$ is a strictly increasing function of $\mu$ due to the properties of the tanfunction. Therefore $\Phi_{1}(\mu)$ is a strictly decreasing function of $\mu$. Since $\Phi_{1}(0)=0$, we get $\Phi_{1}(\mu)<0$ for $\lambda \kappa>\mu>0$ and in view of

$$
\left(\sqrt{\lambda \kappa-\mu} k_{c}+\beta k_{s}\right)\left(\sqrt{\lambda+\mu} h_{c}+\beta h_{s}\right)>0
$$


we obtain $\Phi(\mu)<0$ for $\lambda \kappa>\mu>0$. Note that (48) is always well-defined since $\mu>0$. The sign of $\Phi(\mu)$ and $C_{6}$ will be used in the following section to determine the sign of $I[g]$.

4.2. [Case I: $\mu<\lambda \kappa]$, Computation of $I[g]$. We may write $I[g]$ as

$$
I[g]=\left(P(c)+\varphi^{\prime}(c)\right) \int_{D} g^{2}(y) d y
$$

Assuming $\mu<\lambda \kappa$, we have

$$
P(c)+\varphi^{\prime}(c)=C_{5} h_{c}-C_{6} h_{s}+\varphi^{\prime}(c)=C_{6}\left(-\beta^{-1} \sqrt{\lambda+\mu} h_{c}-h_{s}\right)+\varphi^{\prime}(c) .
$$

The function $\varphi$ is given by (16) with $a=0$, i.e. we have

$$
\varphi(x)=\left\{\begin{array}{lll}
\widetilde{C}_{3} \cos \sqrt{\lambda \kappa} x+\widetilde{C}_{4} \sin \sqrt{\lambda \kappa} x & \text { in } \quad(0, c), \\
\widetilde{C}_{5} \cosh \sqrt{\lambda}(x-1)+\widetilde{C}_{6} \sinh \sqrt{\lambda}(x-1) & \text { in } \quad(c, 1) .
\end{array}\right.
$$

Using boundary and transmission conditions (13)-(15) at $x=c$ we obtain

$$
\begin{aligned}
\varphi(c) & =\widetilde{C}_{4}\left(\beta^{-1} \sqrt{\lambda \kappa} \cos \sqrt{\lambda \kappa} c+\sin \sqrt{\lambda \kappa} c\right)=\widetilde{C}_{4}\left(\beta^{-1} \sqrt{\lambda \kappa} l_{c}+l_{s}\right), \\
\varphi^{\prime}(c) & =\widetilde{C}_{4}\left(-\beta^{-1} \lambda \kappa \sin \sqrt{\lambda \kappa} c+\sqrt{\lambda \kappa} \cos \sqrt{\lambda \kappa} c\right)=\widetilde{C}_{4}\left(-\beta^{-1} \lambda \kappa l_{s}+\sqrt{\lambda \kappa} l_{c}\right),
\end{aligned}
$$

where $\widetilde{C}_{4}$ is a constant which depends on the normalization chosen for $\varphi$. Note that $\widetilde{C}_{4}>0$ since $\lambda$ is a principal eigenvalue. Hence, $P(c)+\varphi^{\prime}(c)>0$ (i.e. $I[g]>0$ ) if

$$
-C_{6}\left(\beta^{-1} \sqrt{\lambda+\mu} h_{c}+h_{s}\right)>-\varphi^{\prime}(c) \text {. }
$$

We have $-C_{6}>0$ and $\beta^{-1} \sqrt{\lambda+\mu} h_{c}+h_{s}>0$. Therefore, if $-\varphi^{\prime}(c) \leq 0$, inequality (53) holds true. Thus, we study the sign of $-\varphi^{\prime}(c)$ as a function of $\beta$.

$$
-\varphi^{\prime}(c)=0 \Longleftrightarrow \widetilde{C}_{4}\left(\beta^{-1} \lambda \kappa \sin \sqrt{\lambda \kappa} c-\sqrt{\lambda \kappa} \cos \sqrt{\lambda \kappa} c\right)=0 \Longleftrightarrow \beta=\sqrt{\lambda \kappa} \tan \sqrt{\lambda \kappa} c .
$$

Clearly, $-\varphi^{\prime}(c)$ is also a decreasing function of $\beta$. Therefore, setting

$$
\hat{\beta}=\sqrt{\lambda \kappa} \tan \sqrt{\lambda \kappa} c,
$$

we have $-\varphi^{\prime}(c)<0$ for $\beta>\hat{\beta},-\varphi^{\prime}(c)=0$ for $\beta=\hat{\beta}$ and $-\varphi^{\prime}(c)>0$ for $\beta<\hat{\beta}$. Thus, (53) holds true for $\beta \geq \hat{\beta}$. Next we study the case $\beta<\hat{\beta}$. The latter implies $-\varphi^{\prime}(c)>0$. Dividing by $-\varphi^{\prime}(c)$ in (53) and using (48) yields

$$
\frac{\lambda(\kappa+1) \varphi(c)}{-\varphi^{\prime}(c)}>\frac{-\Phi(\mu)}{\left(\sqrt{\lambda \kappa-\mu} k_{c}+\beta k_{s}\right)\left(\sqrt{\lambda+\mu} h_{c}+\beta h_{s}\right)} .
$$

Inserting (51)-(52) and the expression for $\Phi(\mu)$ we find that (53) is equivalent to

$$
g_{1}(\beta)>g_{2}(\beta, \mu)+g_{3}(\beta, \mu) .
$$

with

$$
\begin{aligned}
g_{1}(\beta) & =\lambda(\kappa+1) \frac{\sqrt{\lambda \kappa} l_{c}+\beta l_{s}}{\lambda \kappa l_{s}-\beta \sqrt{\lambda \kappa} l_{c}}, \quad g_{2}(\beta, \mu)=\frac{(\lambda+\mu) h_{s}+\beta \sqrt{\lambda+\mu} h_{c}}{\sqrt{\lambda+\mu} h_{c}+\beta h_{s}}, \\
g_{3}(\beta, \mu) & =\frac{-(\lambda \kappa-\mu) k_{s}+\beta \sqrt{\lambda \kappa-\mu} k_{c}}{\sqrt{\lambda \kappa-\mu} k_{c}+\beta k_{s}} .
\end{aligned}
$$


We study now the behaviour of $g_{1}, g_{2}$ and $g_{3}$ as functions of $\beta$. Differentiating with respect to $\beta$ yields

$$
\begin{aligned}
\partial_{\beta} g_{1}(\beta) & =\lambda(\kappa+1) \frac{\lambda \kappa}{\left(\lambda \kappa l_{s}-\beta \sqrt{\lambda \kappa} l_{c}\right)^{2}}>0, \quad \partial_{\beta} g_{2}(\beta, \mu)=\frac{\lambda+\mu}{\left(\sqrt{\lambda+\mu} h_{c}+\beta h_{s}\right)^{2}}>0, \\
\partial_{\beta} g_{3}(\beta, \mu) & =\frac{\lambda \kappa-\mu}{\left(\sqrt{\lambda \kappa-\mu} k_{c}+\beta k_{s}\right)^{2}}>0 .
\end{aligned}
$$

Thus, $g_{1}, g_{2}$ and $g_{3}$ are increasing functions of $\beta$. We have the estimates

$$
\begin{aligned}
g_{2}(\beta, \mu) & =\frac{(\lambda+\mu) h_{s}+\beta \sqrt{\lambda+\mu} h_{c}}{\sqrt{\lambda+\mu} h_{c}+\beta h_{s}} \\
& \leq \frac{(\lambda+\mu) h_{s}+\beta \sqrt{\lambda+\mu} h_{c}}{\sqrt{\lambda+\mu} h_{c}}=\sqrt{\lambda+\mu} \tanh \sqrt{\lambda+\mu}(1-c)+\beta .
\end{aligned}
$$

and further $g_{3}(\beta, \mu) \leq-\sqrt{\lambda \kappa-\mu} \tan \sqrt{\lambda \kappa-\mu} c+\beta$. Since $-\sqrt{\lambda \kappa-\mu} \tan \sqrt{\lambda \kappa-\mu} c<0$ and $\mu<\lambda \kappa$ we get

$$
g_{2}(\beta, \mu)+g_{3}(\beta, \mu) \leq \sqrt{\lambda+\mu} \tanh \sqrt{\lambda+\mu}(1-c)+2 \beta \leq \sqrt{\lambda(1+\kappa)}+2 \beta .
$$

Simplifying $g_{1}(\beta, \mu)$ results in

$$
\begin{aligned}
g_{1}(\beta) & =\lambda(\kappa+1) \frac{\sqrt{\lambda \kappa} l_{c}+\beta l_{s}}{\lambda \kappa l_{s}-\beta \sqrt{\lambda \kappa} l_{c}}=\lambda(\kappa+1) \frac{1+\beta(\sqrt{\lambda \kappa})^{-1} \tan \sqrt{\lambda \kappa} c}{-\beta+\sqrt{\lambda \kappa} \tan \sqrt{\lambda \kappa} c} \\
& =\lambda(\kappa+1) \frac{1+\beta \hat{\beta}(\lambda \kappa)^{-1}}{-\beta+\hat{\beta}} .
\end{aligned}
$$

Based on this expression of $g_{1}$, we now show that

$$
g_{1}(\beta) \geq \sqrt{\lambda(1+\kappa)}+2 \hat{\beta} \geq g_{2}(\beta, \mu)+g_{3}(\beta, \mu) .
$$

For this purpose, we first look for a solution to the equation $g_{1}(\beta)=\sqrt{\lambda(1+\kappa)}+2 \hat{\beta}$. A few transformations lead to

$$
\beta=\frac{\hat{\beta}(2 \hat{\beta}-\sqrt{\lambda(1+\kappa)})-\lambda(1+\kappa)}{\hat{\beta} \frac{1+\kappa}{\kappa}+2 \hat{\beta}+\sqrt{\lambda(1+\kappa)}} .
$$

Using $\hat{\beta}=\sqrt{\lambda \kappa} \tan \sqrt{\lambda \kappa} c$ and (19) we get $\hat{\beta}=\sqrt{\lambda} \tanh \sqrt{\lambda}(1-c) \leq \sqrt{\lambda}$. On one hand, if $2 \hat{\beta}-\sqrt{\lambda(1+\kappa)} \leq 0$, then the solution $\tilde{\beta}$ of (59) satisfies $\tilde{\beta}<0$. On the other hand, if $2 \hat{\beta}-\sqrt{\lambda(1+\kappa)}>0$, we use $\hat{\beta} \leq \sqrt{\lambda}$ and obtain

$$
\tilde{\beta} \leq \frac{\lambda(1-\kappa-\sqrt{1+\kappa})}{\hat{\beta} \frac{1+\kappa}{\kappa}+2 \hat{\beta}+\sqrt{\lambda(1+\kappa)}}<0 .
$$

Therefore, in any case we obtain $\tilde{\beta}<0$. Since $g_{1}(\beta)$ is increasing as a function of $\beta$, we have

$$
g_{1}(\beta) \geq \sqrt{\lambda(1+\kappa)}+2 \hat{\beta} \text { for all } 0<\beta<\hat{\beta},
$$

and since $g_{2}(\beta, \mu), g_{3}(\beta, \mu)$ are also increasing functions of $\beta$ we finally obtain

$$
g_{1}(\beta) \geq \sqrt{\lambda(1+\kappa)}+2 \hat{\beta} \geq g_{2}(\beta, \mu)+g_{3}(\beta, \mu) \text { for all } 0<\beta<\hat{\beta} .
$$


Therefore, (53) and $P(c)+\varphi^{\prime}(c)>0$ (i.e. $I[g]>0$ ) hold true for all $\beta \geq 0$.

4.3. [Case II: $\mu=\lambda \kappa$ ], Computation of $P$. The case $\mu=\lambda \kappa$ is critical as we get $P^{\prime \prime}(x)=0$ for $0<x<c$ in (43), and therefore the solution $P$ is not given by (44), except for $\beta=0$. However, the calculation of $P$ leads to a similar conclusion as for the case $\mu<\lambda \kappa$. In fact, we are looking for a solution of the type

$$
P(x)= \begin{cases}C_{3}+C_{4} x, & 0<x<c, \\ C_{5} \cosh \sqrt{\lambda+\mu}(x-1)+C_{6} \sinh \sqrt{\lambda+\mu}(x-1), & c<x<1,\end{cases}
$$

with constants $C_{i}, i \in\{3,4,5,6\}$. After similar calculations as for the case $\mu<\lambda \kappa$, we end up with the formula

$$
C_{6}=\frac{\lambda(\kappa+1) \varphi(c) \beta(1+\beta c)}{\Phi(\mu)} .
$$

with $\Phi(\mu)=-(1+\beta c)\left((\lambda+\mu) h_{s}+\beta \sqrt{\lambda+\mu} h_{c}\right)-\beta\left(\sqrt{\lambda+\mu} h_{c}+\beta h_{s}\right)<0$. Thus we have $C_{6}<0$ as before. The values of $\varphi(c)$ and $\varphi^{\prime}(c)$ are independent of $\mu$ and are thus, still given by (51)-(52). Hence, we also have $P(c)+\varphi^{\prime}(c)>0$ (i.e. $I[g]>0$ ) if

$$
-C_{6}\left(\beta^{-1} \sqrt{\lambda+\mu} h_{c}+h_{s}\right)>-\varphi^{\prime}(c),
$$

and (63) is always true if $\beta \geq \hat{\beta}$. When $\beta<\hat{\beta}$, (63) can be rewritten as

$$
g_{1}(\beta)>g_{2}(\beta, \mu)+g_{3}(\beta)
$$

where $g_{1}$ and $g_{2}$ are given by $(56)$ and $g_{3}(\beta)=\beta(1+\beta c)^{-1} \leq \beta$. Consequently, we derive the same estimate as (58), i.e.

$$
g_{2}(\beta, \mu)+g_{3}(\beta) \leq \sqrt{\lambda+\mu} \tanh \sqrt{\lambda+\mu}(1-c)+2 \beta \leq \sqrt{\lambda(1+\kappa)}+2 \beta,
$$

and we obtain the same conclusion as in the case $\mu<\lambda \kappa$, i.e. $P(c)+\varphi^{\prime}(c)>0$ (i.e. $I[g]>0$ ) for all $\beta \geq 0$.

4.4. [Case III: $\mu>\lambda \kappa$ ], Computation of $P$. In this case, $P$ is only composed of hyperbolic functions:

$$
P(x)= \begin{cases}C_{3} \cosh \sqrt{\mu-\lambda \kappa} x+C_{4} \sinh \sqrt{\mu-\lambda \kappa} x, & 0<x<c, \\ C_{5} \cosh \sqrt{\lambda+\mu}(x-1)+C_{6} \sinh \sqrt{\lambda+\mu}(x-1), & c<x<1 .\end{cases}
$$

The Robin boundary conditions for $P$ provide

$$
\beta C_{3}=\sqrt{\mu-\lambda \kappa} C_{4}, \quad \beta C_{5}=-\sqrt{\lambda+\mu} C_{6}
$$

From the transmission condition at $x=c$, we have

$$
\begin{aligned}
& C_{5} \sqrt{\lambda+\mu} \sinh (\sqrt{\lambda+\mu}(c-1))+C_{6} \sqrt{\lambda+\mu} \cosh (\sqrt{\lambda+\mu}(c-1)) \\
- & C_{3} \sqrt{\mu-\lambda \kappa} \sinh (\sqrt{\mu-\lambda \kappa} c)-C_{4} \sqrt{\mu-\lambda \kappa} \cosh (\sqrt{\mu-\lambda \kappa} c)=-\lambda(\kappa+1) \varphi(c) \\
& C_{3} \cosh (\sqrt{\mu-\lambda \kappa} c)+C_{4} \sinh (\sqrt{\mu-\lambda \kappa} c) \\
= & C_{5} \cosh (\sqrt{\lambda+\mu}(c-1))+C_{6} \sinh (\sqrt{\lambda+\mu}(c-1))
\end{aligned}
$$


For simplicity, set

$$
\begin{array}{ll}
h_{c}:=\cosh (\sqrt{\lambda+\mu}(1-c)), & h_{s}:=\sinh (\sqrt{\lambda+\mu}(1-c)), \\
k_{c}:=\cosh (\sqrt{\mu-\lambda \kappa} c), & k_{s}:=\sinh (\sqrt{\mu-\lambda \kappa} c) .
\end{array}
$$

Notice that $h_{s}=-\sinh (\sqrt{\lambda+\mu}(c-1))$. Using (66)-(68) we find

$$
C_{6}=\frac{\lambda(\kappa+1) \varphi(c) \beta\left(\sqrt{\mu-\lambda \kappa} k_{c}+\beta k_{s}\right)}{\Phi(\mu)},
$$

with

$$
\begin{aligned}
\Phi(\mu)= & -\left(\sqrt{\mu-\lambda \kappa} k_{c}+\beta k_{s}\right)\left((\lambda+\mu) h_{s}+\beta \sqrt{\lambda+\mu} h_{c}\right) \\
& -\left((\mu-\lambda \kappa) k_{s}+\beta \sqrt{\mu-\lambda \kappa} k_{c}\right)\left(\sqrt{\lambda+\mu} h_{c}+\beta h_{s}\right) .
\end{aligned}
$$

We observe that $\Phi(\mu)<0$ for $\mu>\lambda \kappa$ and $\Phi(\lambda \kappa)=0$. This also implies $C_{6}<0$ for $\mu>\lambda \kappa$. We now use the knowledge of the sign of $C_{6}$ to determine the sign of $I[g]$.

4.5. [Case III: $\mu>\lambda \kappa]$, Computation of $I[g]$. First observe that

$$
I[g]=\left(P(c)+\varphi^{\prime}(c)\right) \int_{D} g^{2}(y) d y
$$

For $\mu>\lambda \kappa$, we have

$$
P(c)+\varphi^{\prime}(c)=C_{5} h_{c}-C_{6} h_{s}+\varphi^{\prime}(c)=C_{6}\left(-\beta^{-1} \sqrt{\lambda+\mu} h_{c}-h_{s}\right)+\varphi^{\prime}(c) .
$$

The values of $\varphi(c)$ and $\varphi^{\prime}(c)$ are given by (51) and (52), respectively. Hence, $P(c)+\varphi^{\prime}(c)>0$ (i.e. $I[g]>0$ ) whenever

$$
-C_{6}\left(\beta^{-1} \sqrt{\lambda+\mu} h_{c}+h_{s}\right)>-\varphi^{\prime}(c) .
$$

As in the case $\mu<\lambda \kappa$, we have that $-C_{6}$ and $\beta^{-1} \sqrt{\lambda+\mu} h_{c}+h_{s}$ are positive. Therefore, if $-\varphi^{\prime}(c)<0$, inequality $(71)$ holds true. Consequently, we draw the same conclusion as before, i.e. (71) is satisfied for $\beta \geq \hat{\beta}$. Next we study the case $\beta<\hat{\beta}$, which implies $-\varphi^{\prime}(c)>0$. Dividing by $-\varphi^{\prime}(c)$ in $(71)$ and using (69) yields

$$
\frac{\lambda(\kappa+1) \varphi(c)}{-\varphi^{\prime}(c)}>\frac{-\Phi(\mu)}{\left(\sqrt{\mu-\lambda \kappa} k_{c}+\beta k_{s}\right)\left(\sqrt{\lambda+\mu} h_{c}+\beta h_{s}\right)} .
$$

Inserting (51)-(52) and the expression of $\Phi(\mu)$ we obtain that (53) is equivalent to

$$
g_{1}(\beta)>g_{2}(\beta, \mu)+g_{3}(\beta, \mu) .
$$

with

$$
\begin{aligned}
g_{1}(\beta) & =\lambda(\kappa+1) \frac{\sqrt{\lambda \kappa} l_{c}+\beta l_{s}}{\lambda \kappa l_{s}-\beta \sqrt{\lambda \kappa} l_{c}}, \quad g_{2}(\beta, \mu)=\frac{(\lambda+\mu) h_{s}+\beta \sqrt{\lambda+\mu} h_{c}}{\sqrt{\lambda+\mu} h_{c}+\beta h_{s}}, \\
g_{3}(\beta, \mu) & =\frac{(\mu-\lambda \kappa) k_{s}+\beta \sqrt{\mu-\lambda \kappa} k_{c}}{\sqrt{\mu-\lambda \kappa} k_{c}+\beta k_{s}} .
\end{aligned}
$$


Next we show that $g_{2}$ and $g_{3}$ are increasing functions of $\mu$. Since $g_{1}$ does not depend on $\mu$, equality (72) occurs for some $\mu_{c}$. Further, we may rewrite $g_{2}$ and $g_{3}$ as

$$
g_{2}(\beta, \mu)=\frac{\sqrt{\lambda+\mu} \tanh \sqrt{\lambda+\mu}(1-c)+\beta}{1+\frac{\beta}{\sqrt{\lambda+\mu}} \tanh \sqrt{\lambda+\mu}(1-c)}, \quad g_{3}(\beta, \mu)=\frac{\sqrt{\mu-\lambda \kappa} \tanh \sqrt{\mu-\lambda \kappa} c+\beta}{1+\frac{\beta}{\sqrt{\mu-\lambda \kappa}} \tanh \sqrt{\mu-\lambda \kappa} c} .
$$

As before, we conclude that $g_{2}$ and $g_{3}$ are increasing functions of $\mu$ and

$$
\lim _{\mu \rightarrow \infty}\left(g_{2}(\beta, \mu)+g_{3}(\beta, \mu)\right)=+\infty \text { for all } 0 \leq \beta<\hat{\beta} .
$$

Consequently, for $\beta<\hat{\beta}$ there exists a unique $\mu_{c}=\mu_{c}(\beta)$ such that $g_{1}(\beta)=g_{2}\left(\beta, \mu_{c}\right)+$ $g_{3}\left(\beta, \mu_{c}\right)$, or, in other words, the equation for $\mu_{c}(\beta)$ is

$$
\begin{aligned}
\lambda(\kappa+1) & \frac{\sqrt{\lambda \kappa}+\beta \tan \sqrt{\lambda \kappa} c}{\lambda \kappa \tan \sqrt{\lambda \kappa} c-\beta \sqrt{\lambda \kappa}} \\
& =\frac{\sqrt{\lambda+\mu_{c}} \tanh \sqrt{\lambda+\mu_{c}}(1-c)+\beta}{1+\frac{\beta}{\sqrt{\lambda+\mu_{c}}} \tanh \sqrt{\lambda+\mu_{c}}(1-c)}+\frac{\sqrt{\mu_{c}-\lambda \kappa} \tanh \sqrt{\mu_{c}-\lambda \kappa} c+\beta}{1+\frac{\beta}{\sqrt{\mu_{c}-\lambda \kappa}} \tanh \sqrt{\mu_{c}-\lambda \kappa} c}
\end{aligned}
$$

Note that for $\beta=0$ we get the equation for the Neumann case

$$
\begin{aligned}
\sqrt{-\lambda \kappa+\mu_{c}} \tanh \sqrt{-\lambda \kappa+\mu_{c}} c+\sqrt{\lambda+\mu_{c}} \tanh \sqrt{\lambda+\mu_{c}}(1-c) \\
=(\kappa+1) \sqrt{\lambda / \kappa} \cot \sqrt{\lambda \kappa} c
\end{aligned}
$$

compare [17]. Therefore in [Case III: $\mu>\lambda \kappa$ ], we conclude:

$$
I[g] \begin{cases}<0 & \text { if } 0 \leq \beta<\hat{\beta} \text { and } \mu>\mu_{c}, \\ =0 & \text { if } 0 \leq \beta<\hat{\beta} \text { and } \mu=\mu_{c}, \\ >0 & \text { if } 0 \leq \beta<\hat{\beta} \text { and } \lambda \kappa<\mu<\mu_{c} . \\ >0 & \text { if } \beta \geq \hat{\beta} .\end{cases}
$$

Summarizing all cases studied above, we obtain the following lemma.

Lemma 1. Let $\mu$ be the smallest positive eigenvalue of (42), and let $\hat{\beta}, \mu_{c}$ be the numbers defined by (54), (73), respectively. If $\left(\mu<\mu_{c}\right.$ and $\left.\beta<\hat{\beta}\right)$ or $(\beta \geq \hat{\beta})$, then the number $\lambda_{2}$ defined by (41) satisfies $\lambda_{2}<0$. If $\left(\mu>\mu_{c}\right.$ and $\left.\beta<\hat{\beta}\right)$ then $\lambda_{2}>0$ and if $\left(\mu=\mu_{c}\right.$ and $\beta<\hat{\beta}$ ) then $\lambda_{2}=0$.

Remark 2. In one dimension, we have observed that there exists a certain $\beta^{*}$ such that for $\beta<\beta^{*}$ the optimal $m$ is the same as for the Neumann problem, while for $\beta>\beta^{*}$ the optimal $m$ is the same as for the Dirichlet problem. In two dimensions, Lemma 1 indicates that we have a similar phenomenon. For $\beta<\hat{\beta}$, the conclusion is similar to the Neumann case [17], i.e. for $\mu<\mu_{c}$ the strip is not optimal, but for $\mu \geq \mu_{c}$ it may be optimal, whereas for $\beta \geq \hat{\beta}$ it is never optimal. Indeed, for $\beta \geq \hat{\beta}$, it is probable that the behaviour is similar to the Dirichlet problem and therefore the solution is located rather in the center of the domain and does not touch the boundary. 


\section{Non-Optimality CASE OF The STRIP $\Omega_{0}^{+}$}

In this section we show that the "strip" $\Omega_{0}^{+}$is not locally optimal when $\lambda_{2}<0$. For this purpose, we use the following characterization of the positive principal eigenvalue [4].

Lemma 2. The positive principal eigenvalue $\lambda$ of (1) is given by the minimum of the Rayleigh quotient

$$
\lambda=\inf _{\psi \in \mathcal{S}(m)} \frac{\int_{\Omega}|\nabla \psi|^{2}+\beta \int_{\partial \Omega} \psi^{2} d s}{\int_{\Omega} m \psi^{2}}
$$

where $\mathcal{S}(m):=\left\{\psi \in H^{1}(\Omega): \int_{\Omega} m \psi^{2}>0\right\}$. Moreover, $\lambda$ is simple, and the infimum is attained only by associated eigenfunctions that do not change sign in $\bar{\Omega}$.

Considering (75) with $m_{\varepsilon}$ instead of $m$, where $m$ corresponds to the aforementioned "strip", and choosing an appropriate feasible point $\psi \in \mathcal{S}\left(m_{\varepsilon}\right)$ we show that $\lambda\left(m_{\varepsilon}\right)<\lambda(m)$ and thus that the strip is not the optimal distribution in this case. Our main result is the following:

Theorem 3. If $\left(\mu<\mu_{c}\right.$ and $\left.\beta<\hat{\beta}\right)$ or $(\beta \geq \hat{\beta})$, then the eigenvalue $\lambda$ corresponding to $m=\kappa \chi_{E}-\chi_{\Omega \backslash E}$ and $\Omega$ given by (32), is not locally optimal.

Proof. Using Lemma 2, we compare $\lambda$ and $\lambda_{\varepsilon}$. For this purpose, we define the functional

$$
J_{\varepsilon}[\psi]:=-\int_{\Omega}|\nabla \psi|^{2}-\beta \int_{\partial \Omega} \psi^{2} d s+\lambda \int_{\Omega} m_{\varepsilon}(x) \psi^{2}
$$

and show that $J_{\varepsilon}[\psi]>0$ for some appropriate $\psi \in \mathcal{S}\left(m_{\varepsilon}\right)$ and small $\varepsilon>0$. In the argument in section $3, \varphi_{2}$ does not play any role in determining $\lambda_{2}$. Hence we choose $\psi=\varphi+\varepsilon \varphi_{1}$ as a test function, where $\varphi_{1}$ is the solution of (38)-(40) constructed in Subsection 4.1.

Since $\varphi \in \mathcal{S}(m)$, we have $\psi \in \mathcal{S}\left(m_{\varepsilon}\right)$ by continuity for sufficiently small $\varepsilon$ and

$$
J_{\varepsilon}\left[\varphi+\varepsilon \varphi_{1}\right]=-\int_{\Omega}\left|\nabla\left(\varphi+\varepsilon \varphi_{1}\right)\right|^{2}-\beta \int_{\partial \Omega}\left(\varphi+\varepsilon \varphi_{1}\right)^{2} d s+\lambda \int_{\Omega} m_{\varepsilon}(x)\left(\varphi+\varepsilon \varphi_{1}\right)^{2} .
$$

The first and the second term are written as

$$
\begin{aligned}
& \int_{\Omega}\left|\nabla\left(\varphi+\varepsilon \varphi_{1}\right)\right|^{2}=\int_{\Omega}|\nabla \varphi|^{2}+2 \varepsilon \int_{\Omega} \nabla \varphi \cdot \nabla \varphi_{1}+\varepsilon^{2} \int_{\Omega}\left|\nabla \varphi_{1}\right|^{2} \\
& \int_{\partial \Omega}\left(\varphi+\varepsilon \varphi_{1}\right)^{2} d s=\int_{\partial \Omega} \varphi^{2} d s+2 \varepsilon \int_{\partial \Omega} \varphi \varphi_{1}+\varepsilon^{2} \varphi_{1}^{2} d s .
\end{aligned}
$$


Using the definition of $m_{\varepsilon}$, we obtain

$$
\begin{aligned}
& \int_{\Omega} m_{\varepsilon}\left(\varphi+\varepsilon \varphi_{1}\right)^{2}=\int_{\Omega} m \varphi\left(\varphi+\varepsilon \varphi_{1}\right) \\
&+\varepsilon\left[\int_{\Omega} m \varphi_{1}\left(\varphi+\varepsilon \varphi_{1}\right)+(\kappa+1) \int_{D} \varphi(c)\left(\varphi(c)+\varepsilon \varphi_{1}(c, y)\right) g(y) d y\right] \\
&+\varepsilon^{2}\left[(\kappa+1) \int_{D}\left\{\varphi_{1}(c, y) \varphi(c) g(y)+\left.\frac{\partial}{\partial x}\left(\varphi^{2}\right)\right|_{x=c} \cdot \frac{g^{2}(y)}{2}\right\} d y\right]+O\left(\varepsilon^{3}\right) \\
&=\int_{\Omega} m \varphi^{2}+\varepsilon {\left[2 \int_{\Omega} m \varphi_{1}+(\kappa+1) \varphi(c)^{2} \int_{D} g(y) d y\right] } \\
&+\varepsilon^{2}\left[\int_{\Omega} m \varphi_{1}^{2}+(\kappa+1) \int_{D} \varphi(c) \varphi_{1}(c, y) g(y) d y\right.\left.+(\kappa+1) \varphi(c) \int_{D}\left\{\varphi_{1}(c, y) g(y)+\varphi^{\prime}(c) g(y)^{2}\right\}\right]+O\left(\varepsilon^{3}\right) .
\end{aligned}
$$

Hence, using (34) and (41) we obtain

$$
\begin{aligned}
\int_{\Omega} m_{\varepsilon}\left(\varphi+\varepsilon \varphi_{1}\right)^{2} & =\int_{\Omega} m \varphi^{2}+2 \varepsilon \int_{\Omega} m \varphi \varphi_{1} \\
& +\varepsilon^{2}\left[\int_{\Omega} m \varphi_{1}^{2}+(\kappa+1) \int_{D} \varphi(c) \varphi_{1}(c, y) g(y) d y-\frac{\lambda_{2}}{\lambda} \int_{\Omega} m \varphi^{2}\right]+O\left(\varepsilon^{3}\right) .
\end{aligned}
$$

This shows

$$
\begin{aligned}
J_{\varepsilon}\left[\varphi+\varepsilon \varphi_{1}\right] & =-\int_{\Omega}|\nabla \varphi|^{2}-\beta \int_{\partial \Omega} \varphi^{2} d s+\lambda \int_{\Omega} m \varphi^{2} \\
+ & 2 \varepsilon\left[-\int_{\Omega} \nabla \varphi \cdot \nabla \varphi_{1}-\beta \int_{\partial \Omega} \varphi \varphi_{1} d s+\lambda \int_{\Omega} m \varphi \varphi_{1}\right] \\
+ & \varepsilon^{2}\left[-\int_{\Omega}\left|\nabla \varphi_{1}\right|^{2}-\beta \int_{\partial \Omega} \varphi_{1}^{2} d s+\lambda \int_{\Omega} m \varphi_{1}^{2}+(\kappa+1) \lambda \int_{D} \varphi(c) \varphi_{1}(c, y) g(y) d y-\lambda_{2} \int_{\Omega} m \varphi^{2}\right] \\
+ & O\left(\varepsilon^{3}\right) \\
=\varepsilon^{2} & {\left[-\int_{\Omega}\left|\nabla \varphi_{1}\right|^{2}-\beta \int_{\partial \Omega} \varphi_{1}^{2} d s+\lambda \int_{\Omega} m \varphi_{1}^{2}+(\kappa+1) \lambda \int_{D} \varphi(c) \varphi_{1}(c, y) g(y) d y-\lambda_{2} \int_{\Omega} m \varphi^{2}\right] } \\
& +O\left(\varepsilon^{3}\right) .
\end{aligned}
$$


Here, due to (38), (39) and (40) we have that

$$
\begin{aligned}
\int_{\Omega}\left|\nabla \varphi_{1}\right|^{2}= & \int_{\Omega_{0}^{+}}\left|\nabla \varphi_{1}\right|^{2}+\int_{\Omega_{0}^{-}}\left|\nabla \varphi_{1}\right|^{2} \\
= & -\int_{\Omega_{0}^{+}} \varphi_{1} \Delta \varphi_{1}+\left.\int_{D} \frac{\partial \varphi_{1}}{\partial x}\right|_{x=c-} \varphi_{1}(c, y) d y \\
& -\int_{\Omega_{0}^{-}} \varphi_{1} \Delta \varphi_{1}-\left.\int_{D} \frac{\partial \varphi_{1}}{\partial x}\right|_{x=c+} \varphi_{1}(c, y) d y-\beta \int_{\partial \Omega} \varphi_{1}^{2} d s \\
= & \lambda \int_{\Omega} m \varphi_{1}^{2}+(\kappa+1) \lambda \int_{D} \varphi(c) \varphi_{1}(c, y) g(y) d y .
\end{aligned}
$$

Consequently, we obtain

$$
J_{\varepsilon}\left[\varphi+\varepsilon \varphi_{1}\right]=-\varepsilon^{2} \lambda_{2} \int_{\Omega} m \varphi^{2}+O\left(\varepsilon^{3}\right) .
$$

This, together with $\lambda_{2}<0$ according to Lemma 1 , implies $J_{\varepsilon}\left[\varphi+\varepsilon \varphi_{1}\right]>0$ and in turn $\lambda_{\varepsilon}<\lambda$ for $\varepsilon$ small enough, showing that $\lambda$ is not locally optimal.

\section{Optimality CASE OF The StRip $\Omega_{0}^{+}$}

Now, we assume $\beta<\hat{\beta}$ and $\mu>\mu_{c}$, where $\mu$ is the smallest eigenvalue of (42) and $\mu_{c}$ solves (73). We show that $\lambda$ is locally minimal in the class of functions

$$
\mathcal{G}:=\left\{g \in L^{2}(D): g \text { satisfies }(34)\right\}
$$

Theorem 4. If $\beta<\hat{\beta}$ and $\mu>\mu_{c}$, then the first eigenvalue $\lambda_{\Omega_{0}^{+}}$of (1) is a local minimum in the set of all perturbations $g \in \mathcal{G}$, with the strip $\Omega_{0}^{+}$given by (33).

Proof. Let $\left\{V_{j}\right\}$ be an orthonormal basis which consists of eigenfunctions of (42):

$$
\left\{\begin{array}{l}
\Delta V_{j}+\mu_{j} V_{j}=0 \quad \text { in } D, \\
\frac{\partial}{\partial n_{y}} V_{j}=0 \text { on } \partial D .
\end{array}\right.
$$

In particular, we set $\mu_{0}=0$ and $V_{0}=1 /|D|$. Since $g$ is orthogonal to $V_{0}$ by (34), we expand $g$ as $g=\sum_{j=1}^{\infty} d_{j} V_{j}$. Then $\varphi_{1}$ is computed as $\varphi_{1}(x, y)=\sum_{j=1}^{\infty} d_{j} P_{j}(x) V_{j}(y)$, where $P_{j}$ satisfies

$$
\left\{\begin{array}{l}
P_{j}^{\prime \prime}(x)+\left(\lambda \kappa-\mu_{j}\right) P_{j}(x)=0, \quad 0<x<c, \\
P_{j}^{\prime \prime}(x)-\left(\lambda+\mu_{j}\right) P_{j}(x)=0, \quad c<x<1, \\
P_{j}^{\prime}(0)-\beta P_{j}(0)=P_{j}^{\prime}(1)+\beta P_{j}(1)=0, \\
P_{j}(c+)=P_{j}(c-), \\
P_{j}^{\prime}(c+)-P_{j}^{\prime}(c-)=-\lambda(\kappa+1) \varphi(c) .
\end{array}\right.
$$


We compute

$$
\begin{aligned}
I[g] & =\int_{D} \varphi_{1}(c, y) g(y)+\varphi^{\prime}(c) g^{2}(y) d y \\
& =\int_{D}\left(\sum_{j=1}^{\infty} d_{j} P_{j}(c) V_{j}(y)\right)\left(\sum_{j=1}^{\infty} d_{j} V_{j}(y)\right) d y+\int_{D} \varphi^{\prime}(c)\left(\sum_{j=1}^{\infty} d_{j} V_{j}(y)\right)^{2} d y \\
& =\sum_{j=1}^{\infty} d_{j}^{2} P_{j}(c)+\varphi^{\prime}(c) \sum_{j=1}^{\infty} d_{j}^{2}=\left(P_{j}(c)+\varphi^{\prime}(c)\right) \sum_{j=1}^{\infty} d_{j}^{2},
\end{aligned}
$$

where we use $\int_{D} V_{j}(y) V_{k}(y) d y=1$ if $j=k$ and 0 if $j \neq k$. From section (4.5) we know that $P_{j}(c)+\varphi^{\prime}(c)<0$ if $\mu_{j}>\mu_{c}$ and $\beta<\hat{\beta}$. By assumption, $\mu_{j} \geq \mu>\mu_{c}$ for all $j$ and we obtain $I[g]<0$ as well as $\lambda_{2}>0$. Thus, we have shown that $\lambda_{\varepsilon}>\lambda$ holds for any $g \neq 0$, $g \in L^{2}(D)$, and sufficiently small $\varepsilon \neq 0$.

\section{Numerical RESULTS}

Now we define an algorithm which is based on the variational formulation (75) of the principal eigenvalue. Since it strongly relies on the Rayleigh quotient (75), it is particularly designed for eigenvalue problems and may have limited applicability for more general minimization problems. In contrast to methods relying on shape and topological sensitivities $[10,15,21,24,25]$ it does not make use of derivative or local perturbation based techniques. In our numerical tests it typically converges to the global optimum of the respective problem, although this has not been established theoretically. In special cases it may get stuck at critical points.

7.1. Algorithm. In this section, we are interested in finding an optimal configuration $m$ which minimizes the principle eigenvalue $\lambda$ of (1) and satisfies the constraint (2). According to Theorem 1, we are looking for an optimal configuration $m$ of bang-bang type, i.e.

$$
m(x):=m_{E}(x)=\left\{\begin{array}{cl}
\kappa & \text { if } x \in E, \\
-1 & \text { if } x \in \Omega \backslash E .
\end{array}\right.
$$

Our method utilizes the rearrangement approach proposed in $[11,18,26]$.

We initialize our algorithm by $E_{0} \subset \mathbb{R}^{N}$ with $\left|E_{0}\right|=m_{0}$ and the initial weight $m_{E_{0}}(x):=$ $\kappa$ if $x \in E_{0}$ and $m_{E_{0}}(x):=-1$ if $x \in \Omega \backslash E_{0}$. Let $\left(\varphi_{E_{0}}, \lambda_{E_{0}}\right)$ be the eigenpair associated to (1). Given $h \in \mathbb{R}$, we define the variable domain $E(h):=\left\{x \in \Omega: \varphi_{E_{0}}(x) \geq h\right\}$ and associated volume $z(h)=|E(h)|$. Defining $h_{0}=\min \left\{\varphi_{E_{0}}(x): x \in E_{0}\right\}$, we then have $E_{0} \subset E\left(h_{0}\right)$ and $m_{0} \leq z\left(h_{0}\right)$. The function $z$ is obviously continuous and decreasing with respect to $h$. Therefore there exists $h_{1} \geq h_{0}$ such that $m_{0}=z\left(h_{1}\right) \leq z\left(h_{0}\right)$. Define the new set $E_{1}:=E\left(h_{1}\right)$ and let $\left(\varphi_{E_{1}}, \lambda_{E_{1}}\right)$ be the eigenpair associated to $m_{E_{1}}$. Then we have the following result regarding $E_{1}$.

Lemma 3. The eigenvalues associated with $E_{0}$ and $E_{1}$ satisfy $\lambda_{E_{1}} \leq \lambda_{E_{0}}$. 
Proof. First we prove that $\int_{\Omega} m_{E_{1}} \varphi_{E_{0}}^{2} \geq \int_{\Omega} m_{E_{0}} \varphi_{E_{0}}^{2}$. In fact, we have

$$
\begin{aligned}
\int_{\Omega} m_{E_{1}} \varphi_{E_{0}}^{2}-\int_{\Omega} m_{E_{0}} \varphi_{E_{0}}^{2} & =\int_{\left(E_{1} \backslash E_{0}\right) \cup\left(E_{0} \backslash E_{1}\right)}\left(m_{E_{1}}-m_{E_{0}}\right) \varphi_{E_{0}}^{2} \\
& =(\kappa+1) \int_{E_{1} \backslash E_{0}} \varphi_{E_{0}}^{2}-(\kappa+1) \int_{E_{0} \backslash E_{1}} \varphi_{E_{0}}^{2} \\
& =(\kappa+1)\left(\int_{E_{1} \backslash E_{0}} \varphi_{E_{0}}^{2}-\int_{E_{0} \backslash E_{1}} \varphi_{E_{0}}^{2}\right) \geq 0 .
\end{aligned}
$$

Indeed, in view of the definition of $E_{1}$, for all $x \in E_{0} \backslash E_{1}$ and all $y \in E_{1} \backslash E_{0}$ we have $\varphi_{E_{0}}(y) \geq h_{1}>\varphi_{E_{0}}(x)$. We also use $\left|E_{1} \backslash E_{0}\right|=\left|E_{0} \backslash E_{1}\right|$, which is due to $z\left(h_{1}\right)=m_{0}$, to compare the integrals.

Using the variational formulation (75) we get

$$
\begin{aligned}
\lambda_{E_{0}} & =\frac{\int_{\Omega}\left|\nabla \varphi_{E_{0}}\right|^{2}+\beta \int_{\partial \Omega} \varphi_{E_{0}}^{2} d s}{\int_{\Omega} m_{E_{0}} \varphi_{E_{0}}^{2}} \\
& \geq \frac{\int_{\Omega}\left|\nabla \varphi_{E_{0}}\right|^{2}+\beta \int_{\partial \Omega} \varphi_{E_{0}}^{2} d s}{\int_{\Omega} m_{E_{1}} \varphi_{E_{0}}^{2}} \geq \inf _{\psi \in \mathcal{S}(m)} \frac{\int_{\Omega}|\nabla \psi|^{2}+\beta \int_{\partial \Omega} \psi^{2} d s}{\int_{\Omega} m_{E_{1}} \psi^{2}}=\lambda_{E_{1}}
\end{aligned}
$$

and the proof is complete.

Note that the equality case in Lemma 3 only happens when $E_{0}=E\left(h_{0}\right)$, which is equivalent to $\varphi_{E_{0}}$ being constant on $\partial E_{0}$. This property at the critical point has been proved for Dirichlet boundary conditions in [12].

Remark 3. Our algorithm may be seen as a natural application of the Rayleigh quotient formulation of the eigenvalue. Indeed, the problem of minimizing $\lambda(m)$ with respect to $m$ is a min-min problem due to the Rayleigh quotient (75). Exchanging the two min we may rewrite the problem as

$$
\min _{m \in \mathcal{M}} \lambda(m)=\min _{\psi \in \mathcal{S}(m)} \min _{m \in \mathcal{M}} \frac{\int_{\Omega}|\nabla \psi|^{2}+\beta \int_{\partial \Omega} \psi^{2} d s}{\int_{\Omega} m \psi^{2}} .
$$

For fixed $\psi \in \mathcal{S}(m)$ the minimization with respect to $m$ is equivalent to solving a max problem

$$
\min _{m \in \mathcal{M}} \frac{\int_{\Omega}|\nabla \psi|^{2}+\beta \int_{\partial \Omega} \psi^{2} d s}{\int_{\Omega} m \psi^{2}} \Leftrightarrow \max _{m \in \mathcal{M}} \frac{\int_{\Omega} m \psi^{2}}{\int_{\Omega}|\nabla \psi|^{2}+\beta \int_{\partial \Omega} \psi^{2} d s} .
$$


The solution of the max problem for fixed $\psi$ in (80) is in fact exactly the level set $E\left(h_{1}\right)$ in our algorithm. The minimization with respect to $\psi$ then corresponds to finding the eigenfunction for the new set $E\left(h_{1}\right)$.

At the discrete level, we need to first compute the forward eigenvalue problem (1), i.e. finding the eigenpair $(\lambda, \varphi)$ for given $m$, then look for the rearrangement satisfying Lemma 3 , and then we repeat this procedure until no furthermore rearrangement of $m$ or improvement of the eigenvalue can be obtained. The forward problem is built by expanding the eigenfunction in terms of a finite element basis (i.e., $\varphi=\sum_{i \in \mathcal{I}} \varphi_{i} \xi_{i}$, where $\xi_{i}$ are basis functions and $\mathcal{I} \subset \mathbb{N}$ is a set of indices), multiplying by a basis element, and integrating over the domain $\Omega$. We assume that $m$ is constant in each element, i.e. either $m=-1$ or $m=\kappa$. This leads to a generalized eigenvalue equation which can be solved by the Arnoldi algorithm [3]. Our implementation relies on the Matlab Partial Differential Equation Toolbox utilizing piecewise linear and globally continuous finite elements.

To find a rearrangement satisfying Lemma 3, the integration is approximated by the quadrature rule

$$
\int_{\Omega} m^{(k-1)}(x)\left(\varphi^{(k-1)}(x)\right)^{2} d x \approx \sum_{i \in \mathcal{I}} w_{i} m_{i}^{(k-1)}\left(\bar{\varphi}_{i}^{(k-1)}\right)^{2},
$$

where $\bar{\varphi}_{i}$ represents $\varphi$ evaluated at a barycenter point of an element. In order to reduce effects due to heterogeneous meshes, we use a uniform mesh [18]. In this case, $w_{i}$ is the area of each triangle. In view of Lemma 3 , given the current set of values $\left\{m_{i}^{(k-1)}\right\}_{i}$ we look for a rearrangement $\left\{m_{i}^{(k)}\right\}_{i}$ satisfying

$$
\sum_{i \in \mathcal{I}} m_{i}^{(k)}\left(\bar{\varphi}_{i}^{(k-1)}\right)^{2} \geq \sum_{i \in \mathcal{I}} m_{i}^{(k-1)}\left(\bar{\varphi}_{i}^{(k-1)}\right)^{2} .
$$

The discrete rearrangement inequality [27] states that

$$
x_{n} y_{1}+\ldots+x_{1} y_{n} \leq x_{\sigma(1)} y_{1}+\ldots+x_{\sigma(n)} y_{n} \leq x_{1} y_{1}+\ldots+x_{n} y_{n}
$$

for every choice of real numbers $x_{1} \leq \ldots \leq x_{n}, y_{1} \leq \ldots \leq y_{n}$ and every permutation $x_{\sigma(1)}, \ldots, x_{\sigma(n)}$ of $x_{1}, \ldots, x_{n}$. Thus, if we sort both $\left(\bar{\varphi}_{i}^{(k-1)}\right)^{2}$ and $m_{i}^{(k-1)}$ in ascending order, $m_{i}^{(k)}$ can be generated by rearranging $m_{i}^{(k-1)}$ using (81). In our case, $m_{i}^{k}$ is either -1 or $\kappa$.

7.2. Numerical tests. In this section we denote $\Omega^{+}:=\{x \in \Omega: m(x)>0\}$. We start with simulations in one dimension and consider $\Omega:=(0,1)$. In Figure 1 , we first demonstrate how the principal eigenvalue $\lambda$ varies with respect to $a$ and $\beta$ for $\Omega^{+}=[a, b]$, where $b-a=c$. The parameters are $\kappa=1, c=0.2$. $a=0.01 p$ with $0 \leq p \leq 80, p \in \mathbb{N}$, and $\beta=2^{0.1 q}$ where $0 \leq q \leq 100, q \in \mathbb{N}$. We observe that $\lambda$ is an increasing function of $\beta$ for any fixed $c$. We verify the existence of the threshold $\beta^{*}$ obtained in Theorem 2 such that $\lambda$ achieves its minimum for $a=(1-c) / 2$ when $\beta>\beta^{*}$ and for $a=0$ or $1-c$ when $\beta<\beta^{*}$. 


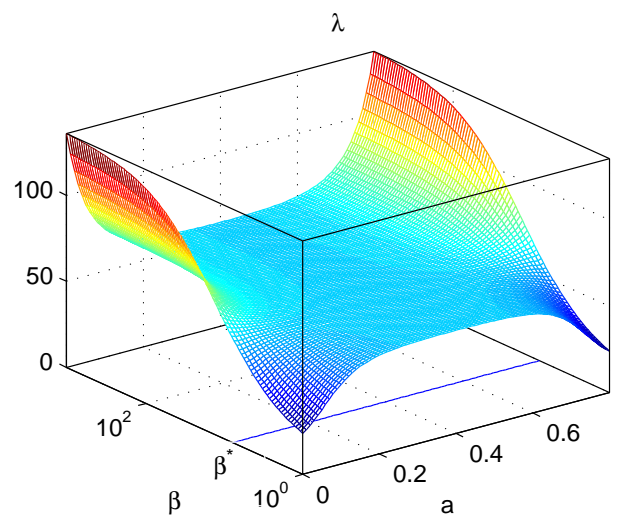

Figure 1. Eigenvalue $\lambda$ versus $a$ and $\beta$ in dimension one. The first eigenvalue $\lambda$ achieves its minimum at $a=0.4$ for $\beta>\beta^{+}$and at $a=0$ or $a=0.8$ for $\beta<\beta^{*}$.

In Figures 2 and 3, we show one-dimensional simulations for $\beta=10$ and $\beta=1$, respectively. The initial condition is chosen as a piecewise constant function

$$
m(x)=\sum_{i=1}^{5}(1+\kappa) H\left(\left|x-x_{i}\right|-0.03\right)-1,
$$

where $H$ is the Heasiviside function, $x_{i}=0.1+0.2 \cdot(i-1)$ for Figure 2 and $x_{i}=0.08+0.2$. $(i-1)$ for Figure 3. In these two cases we have

$$
\mu=-|\Omega|^{-1} \int_{\Omega} m(x) d x=0.4, c=\frac{1-\mu}{1+\kappa}=\frac{0.6}{2}=0.3, \beta^{*}=\frac{\pi}{2 c} \approx \frac{3.14}{2 \cdot 0.3}=5.233 .
$$

Thus we expect the optimal arrangement for $\Omega^{+}$to be at the center of $\Omega$ for $\beta=10>\beta^{*}$ and at the one end of $\Omega=(0,1)$ for $\beta=1<\beta^{*}$.

In Figure 2, we also observe a merging behaviour during the optimization process. The optimal arrangement of $\Omega^{+}$is at the center, i.e. $\Omega^{+}=(0.35,0.65)$, where the minimal eigenvalue $\lambda_{1}=24.0991$ is achieved.

In Figure 3, we depict a situation where the domain $\Omega^{+}$undergoes topological changes during the optimization process. The domain $\Omega^{+}$starts to merge into two pieces from initially five pieces and then it becomes a simply connected component $\Omega^{+}=[0,0.3]$ at the second iteration which is the optimal arrangement with $\lambda=11.3293$. In these two examples, the optimization process is extremely fast in the sense that the optimal arrangements are achieved in just three iterations. These numerical results in one dimension strongly indicate that the optimum corresponds to a simply connected component.

In Figures 4-13, we consider test runs in two dimensions where $\Omega$ is a square. Although this does not coincide with our assumptions that $\Omega$ has to be smooth, one may actually prove that the existence results in section 1 remain true if $\Omega$ is a convex polygonal domain, 

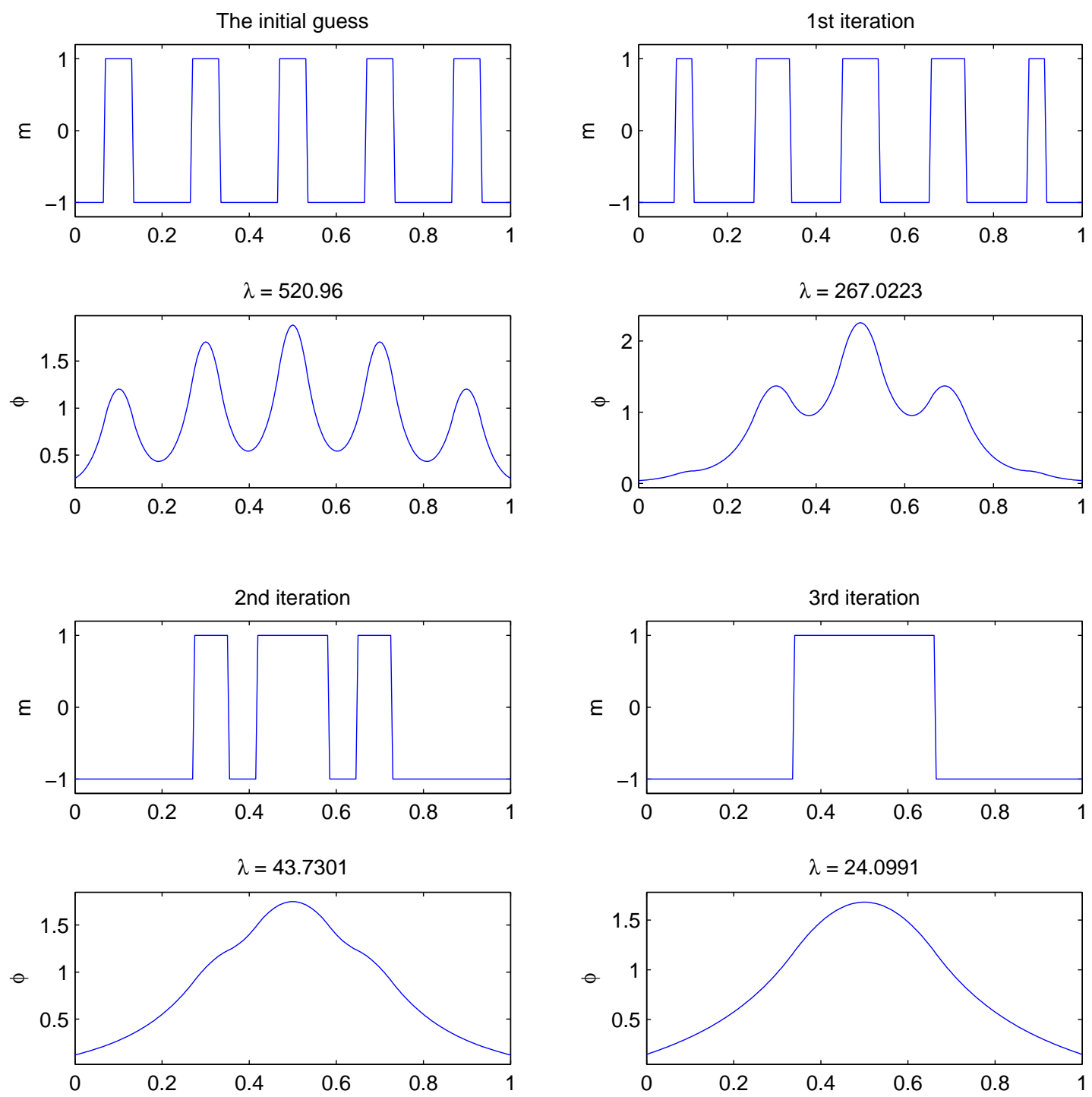

Figure 2. The evolution of $m$ and its corresponding first eigenfunction for $\beta=10$.

according to the solution theory of elliptic equations in non-smooth domains; see [14] for instance. Note that this is no longer true if $\Omega$ has reentrant corners.

The computational domain $[0,1] \times[0,1]$ is first triangulated into four triangles by connecting the four vertices with the point $(0.5,0.5)$ and then further refined 8 times by dividing each triangles into four triangles. The total number of triangles is $4^{9}=262144$. We use 

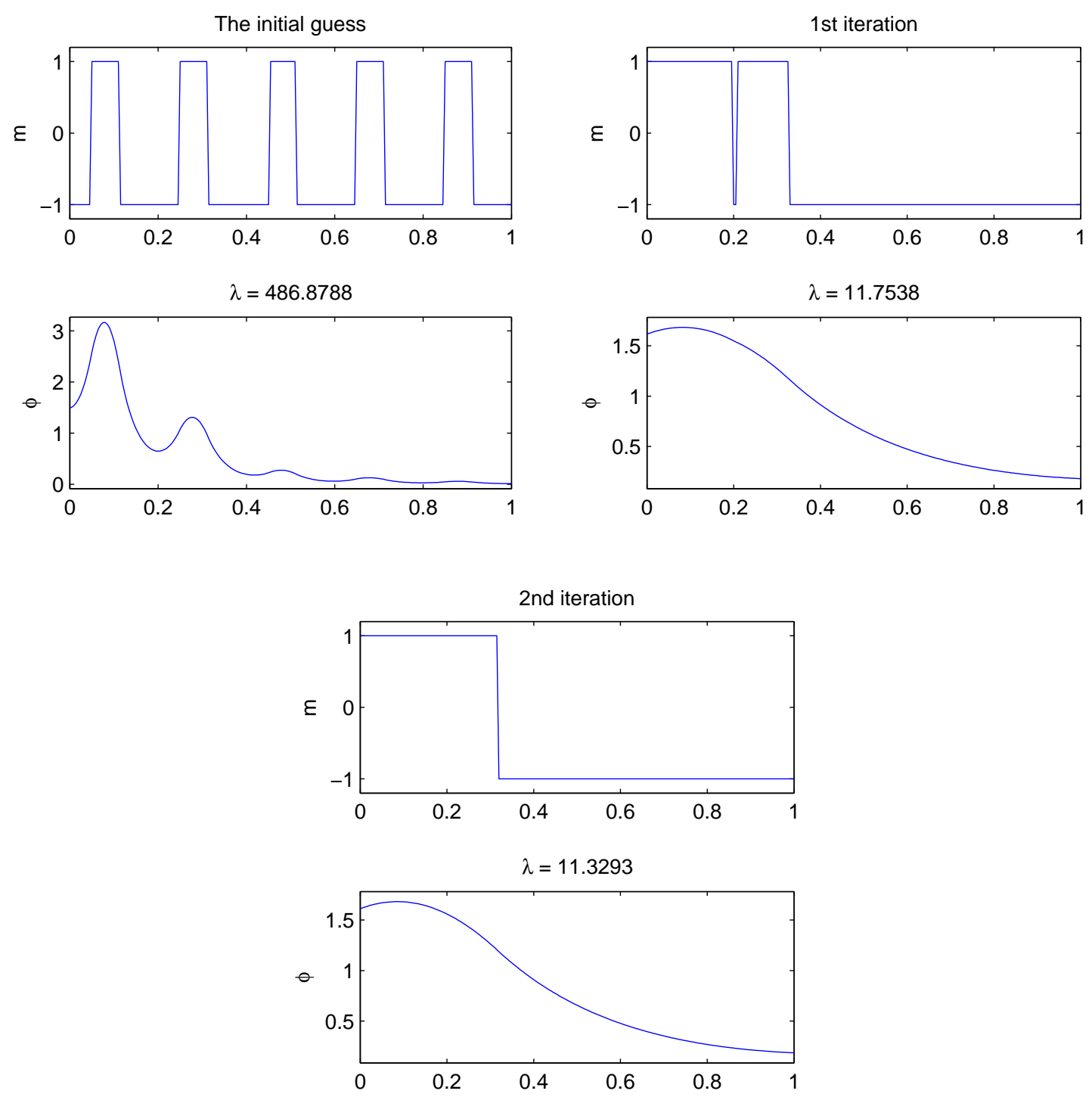

Figure 3. The evolution of $m$ and its corresponding first eigenfunction for $\beta=1$.

linear element and the eigenvalues have second order accuracy. The algorithm is terminated when the $(n-1)$-st and $n$-th iterations yield precisely the same configuration $m$, respectively.

In Figure 4-10, we consider mixed Robin-Neumann conditions for cylindrical domains as in Sections 3-6, i.e. we have Neumann conditions on the top and bottom boundaries and Robin conditions on the side. 
The initial configuration is $m(x, y)=1$ for $\{(x, y) \mid x<0.4+0.15 \sin (4 \pi y)\}$ and $m(x, y)=$ -1 otherwise for Figure 4-6. The configurations of $m(x, y)$ at iteration $0,1,2$, and 47 and the corresponding principal eigenvalue at different iterations are shown in Figure 4 for $\beta=0.1$. We found that the optimal shape for $\Omega^{+}$becomes a strip parallel to the boundary with Robin boundary conditions. The eigenvalue $\lambda_{1}$ decreases very fast in the first few iterations, see the last figure in Figure 4. The algorithm stops only when no furthermore improvement of $m(x, y)$ can be made, which takes 46 iterations in total. However, the deviation of the eigenvalue at iteration 6 , i.e. $\lambda_{1}^{6}$, is already less than 0.005 from the optimal numerical eigenvalue $\lambda_{1}^{(46)}=3.4488$.

In Figures 5-6, we keep the initial condition unchanged but $\beta$ is increased to 1 and 10, respectively. The optimal shape for $\Omega^{+}$with $\beta=1$ is no longer a strip with straight boundaries, rather, it becomes a curved strip at iteration 70, as shown in Figure 5. When $\beta=10$, then the optimal shape for $\Omega^{+}$becomes a half-circle like shape attached to one of the two boundary edges with Neumann condition as shown in Figure 6 .

In Figures 7-9, the initial configuration is $m(x, y)=1$ for $\{(x, y) \mid x<0.25+0.15 \sin (4 \pi y)\}$ and $m(x, y)=-1$ otherwise. The area of $\Omega^{+}$is smaller than in the previous three examples. Notice that the optimal shape becomes a quarter of a circular-like shape at one of the corner for both $\beta=0.1$ and $\beta=1$ in Figures 7-8. For $\beta=10$, the optimal shape is a half-circle like shape as shown in Figure 9. This result is very similar to the one shown in Figure 6.

In general we observe that the final result seems independent of the initial guess, up to the non-uniqueness of the solutions of (6). Indeed, the minimization problem (6) may have several global minima in general, due to possible symmetries of the domain $\Omega$ and of the boundary conditions. If this is the case, as in our examples, the initial guess determines which of these global optima will be selected. This is illustrated in Figure $10\left(a_{1}\right),\left(b_{1}\right),\left(c_{1}\right)$ and $\left(d_{1}\right)$, where four different initial conditions are chosen. The final optimal configurations of $m$ are shown in $\left(a_{2}\right),\left(b_{2}\right),\left(c_{2}\right)$ and $\left(d_{2}\right)$, respectively. The white strip can either be attached to the left or to the right boundary due to the symmetry of the square and to the mixed Robin-Neumann conditions. Both configurations yield the same minimal first eigenvalue. We remark here that some initial choices may lead to local minimal configurations.

In Figure 11-13, we show test runs with Robin conditions on all boundaries for different $\beta$-values. The initial condition is again $m(x, y)=1$ for $\{(x, y) \mid x<0.4+0.15 \sin (4 \pi y)\}$ and $m(x, y)=-1$ otherwise. The optimal shape for $\Omega^{+}$is a curved strip for $\beta=0.1$ (see Figure 11), a quarter of a circular-like shape at a corner for $\beta=1$ (see Figure 12) and a circular shape in the center for $\beta=10$ (see Figure 13), respectively. This behavior is similar to the one-dimensional case, i.e. when $\beta>\beta^{*}$ and the optimal shape for $\Omega^{+}$rests at the center of the domain.

These test runs indicate that the optimal domain for $\Omega^{+}$is most likely simply connected. When the area of $\Omega^{+}$is large enough and $\beta$ is sufficiently small, then a strip parallel to the boundary with Robin boundary conditions is the optimal configuration. Otherwise, for small area $\left|\Omega^{+}\right|$, the optimal shape for $\Omega^{+}$becomes a quarter of a circular-like shape, a half-circular like shape or a curved strip attached to the boundary when $\beta$ is small. When 

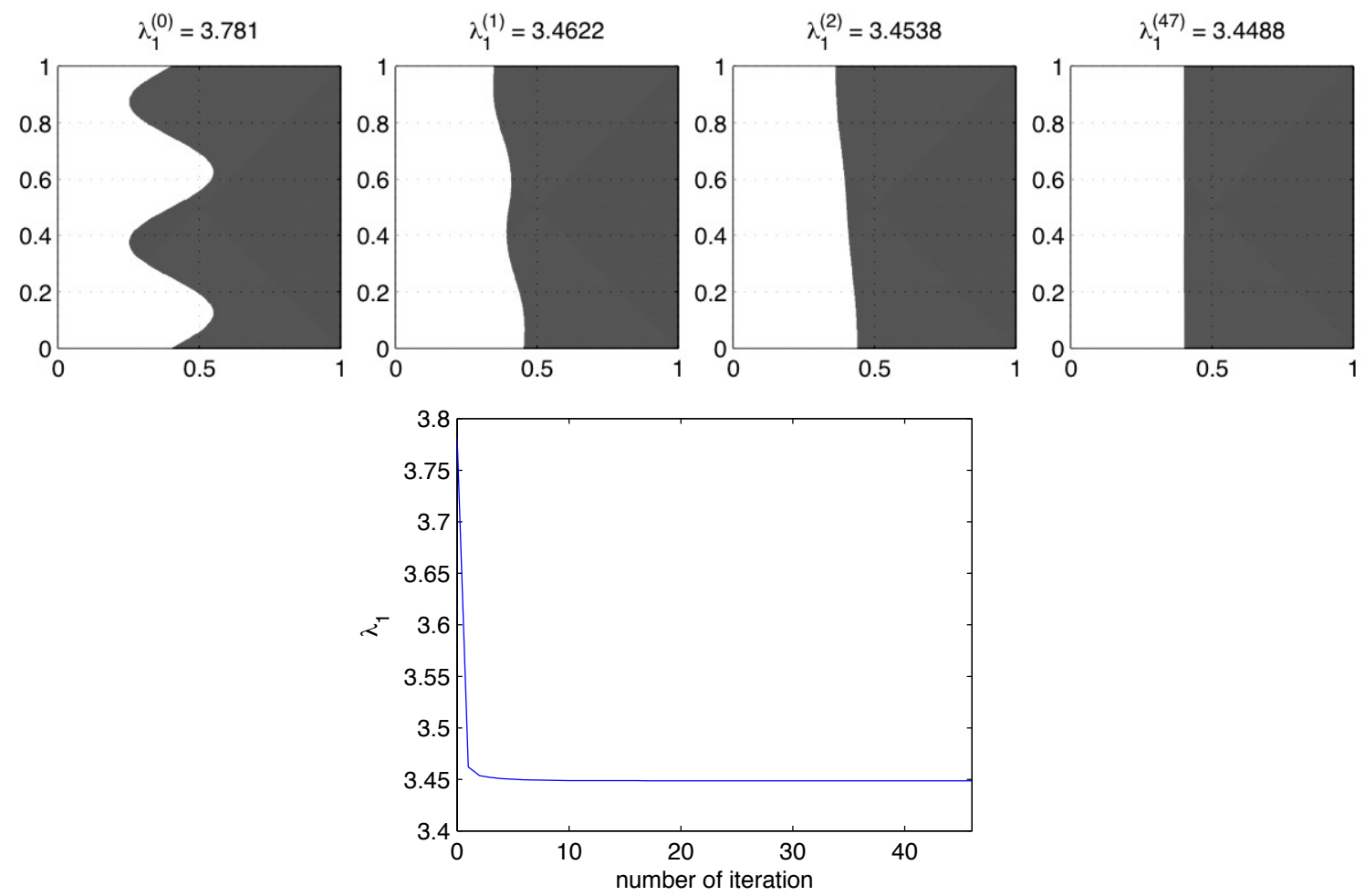

FiguRE 4. Mixed Robin-Neumann conditions with $\beta=0.1$ : the initial configuration is $m(x, y)=1$ for $\{(x, y) \mid x<0.4+0.15 \sin (4 \pi y)\}$ and $m(x, y)=-1$ otherwise. The configurations of $m(x, y)$ at iterations $0,1,2$, and 47 and the corresponding principal eigenvalue at different iterations.

$\beta$ is large, then the optimal shape for $\Omega^{+}$becomes a circular-like shape at the center of the domain.

\section{CONCLUSiON}

In one dimension, we have shown the existence of a threshold value $\beta^{*}$ such that the minimum of the principal eigenvalue, among simply connected sets $E$, is attained when $E$ is on the boundary for $\beta<\beta^{*}$, as in the Neumann case, and when $E$ is in the center for $\beta>\beta^{*}$, as in the Dirichlet case. The case $\beta=\beta^{*}$ is a perfect equilibrium and every set $E$ with one connected component is optimal. The question of optimality for a general set $E$ is still open: numerical results indicate that the eigenvalue is always higher for $E$ with several connected components. This is due to the oscillations of the corresponding eigenfunction. Therefore, we conjecture that the minimal eigenvalue is obtained for $E$ with only one connected component.

In higher dimensions, we expect in general the existence of a similar threshold value $\beta^{*}$. The case of Robin boundary conditions on all of the boundary is difficult to study, 

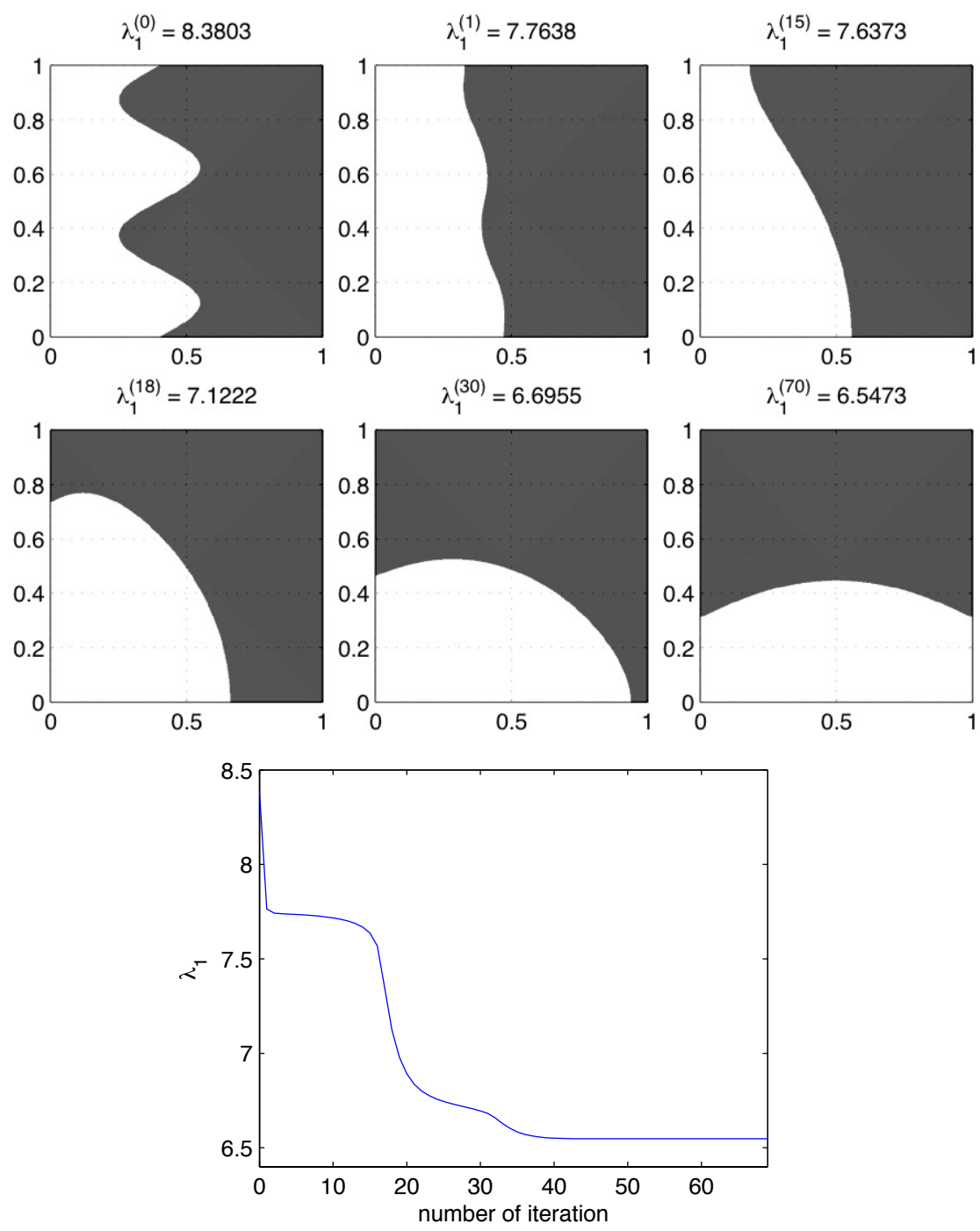

Figure 5. Mixed Robin-Neumann conditions with $\beta=1$ : the initial configuration is $m(x, y)=1$ for $\{(x, y) \mid x<0.4+0.15 \sin (4 \pi y)\}$ and $m(x, y)=-1$ otherwise. The configurations of $m(x, y)$ at iterations $0,1,30$, and 70 and the corresponding principal eigenvalue at different iterations.

since we cannot use any separation of variables to simplify the problem, even with simple geometries such as cylindrical domains. In this paper we have considered mixed RobinNeumann conditions and we have shown that such a threshold $\beta^{*}$ also exists for cylindrical domains. In the favorable case we have shown, that a "strip" touching the boundary of the cylinder is locally optimal. Unlike in dimension one, additional conditions for the optimality 

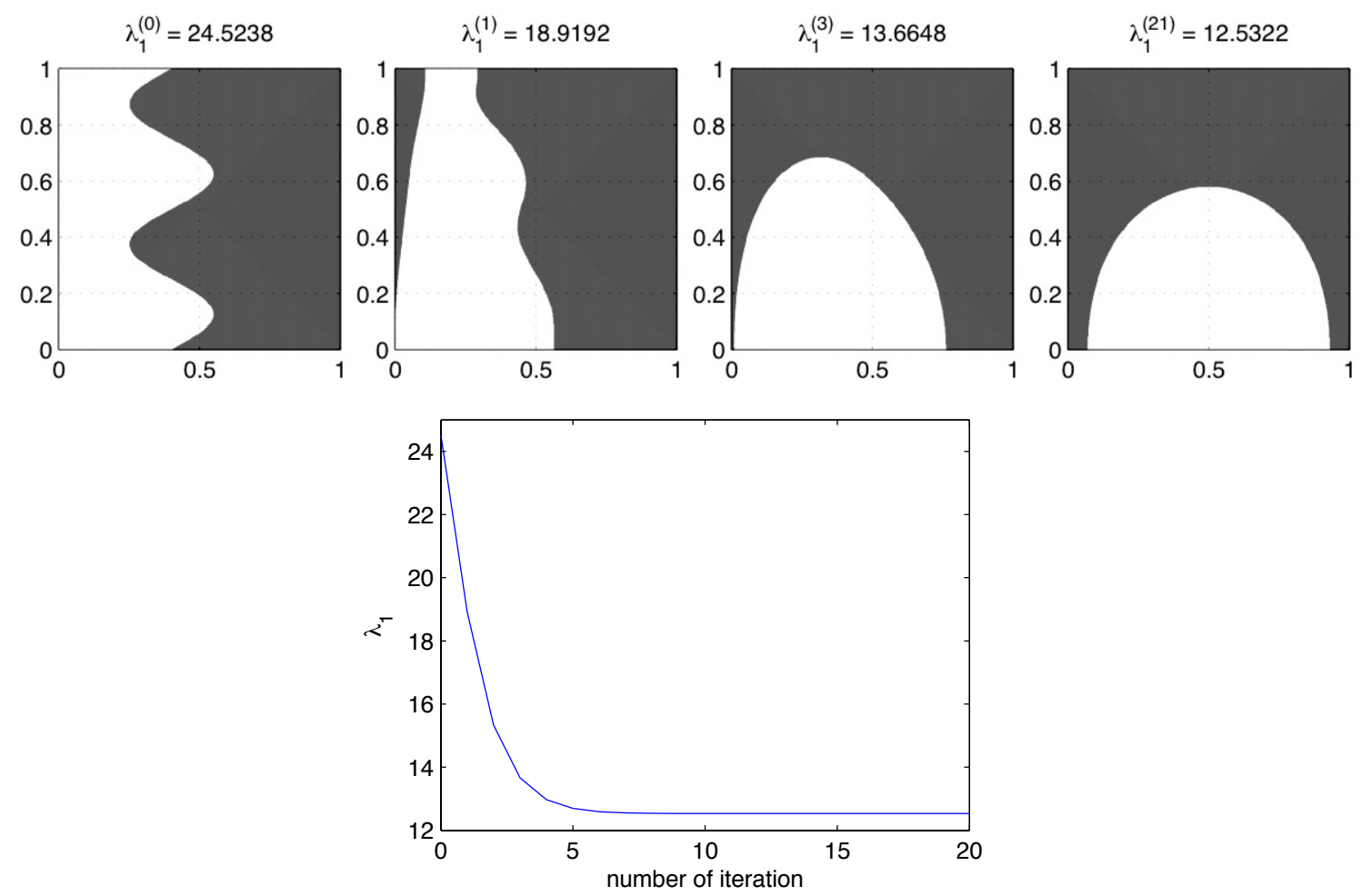

FiguRE 6. Mixed Robin-Neumann conditions with $\beta=10$ : the initial configuration is $m(x, y)=1$ for $\{(x, y) \mid x<0.4+0.15 \sin (4 \pi y)\}$ and $m(x, y)=-1$ otherwise. The configurations of $m(x, y)$ at iterations $0,1,3$, and 21 and the corresponding principal eigenvalue at different iterations.

of the strip come into play. In particular, these conditions depend on the shape and the length of the cylinder.

For multi-dimensional domains and Robin boundary conditions on all of the boundary the optimal set $E$ changes continuously with $\beta$. Therefore an interesting task is to find $\beta^{*}$ such that the optimal $E$ does not touch the boundary for $\beta>\beta^{*}$. Other interesting questions are the study of the case $\beta<0$ and the dependence of $\mu_{c}$ on $\beta$.

\section{ACKNOWLEDGMENT}

Michael Hintermüller and Antoine Laurain are supported by START-Project "Interfaces and Free Boundaries", SFB "Mathematical Optimization and Applications in Biomedical Science", DFG research center MATHEON and SPP1253 "Optimization with PDE's". ChiuYen Kao was partially supported by the National Science Foundation grant DMS-0811003 and Alfred P. Sloan Fellowship. This work was initiated while C.-Y. Kao was a research visitor at the START-Project, University of Graz. 

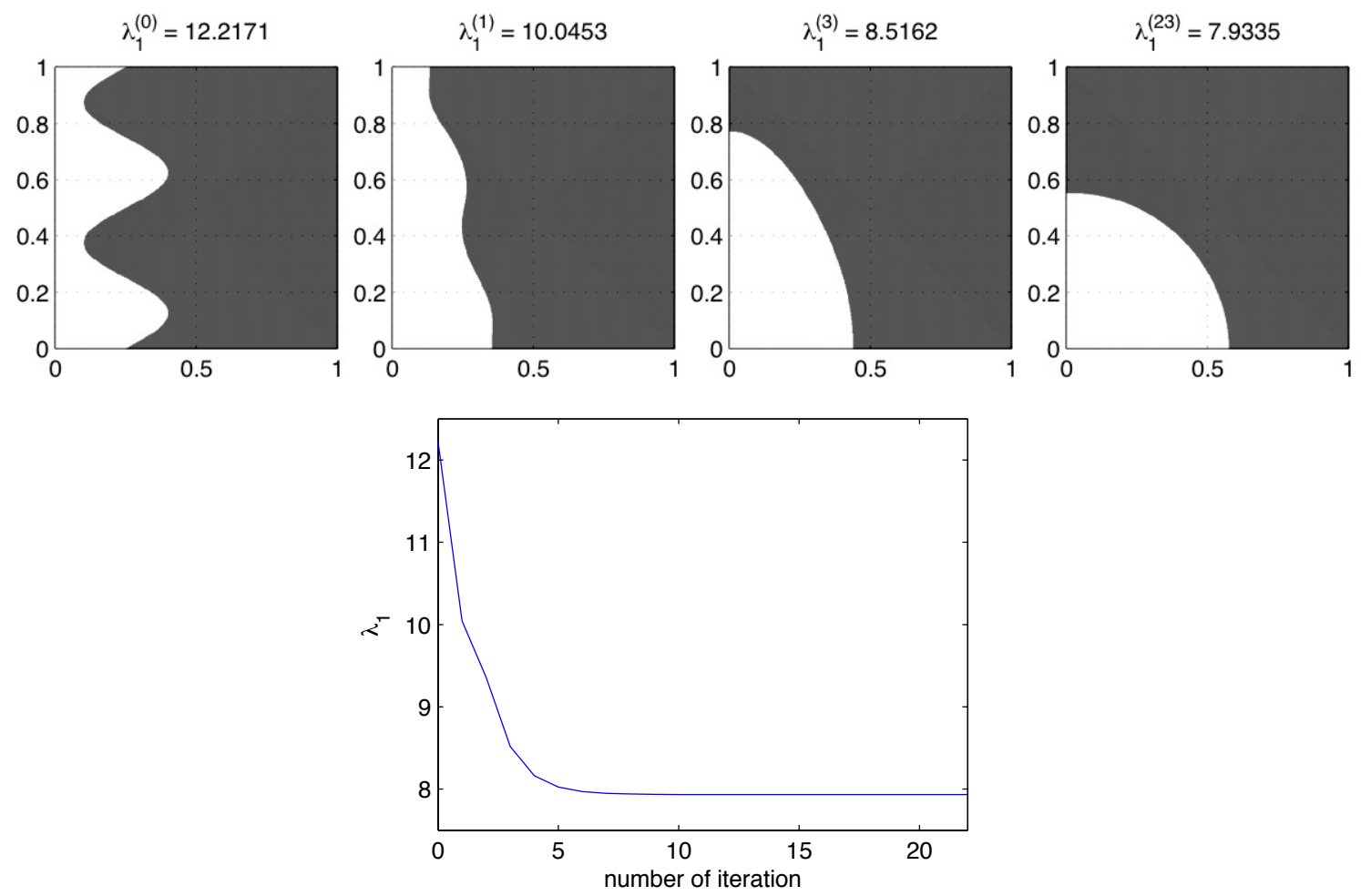

FiguRe 7. Mixed Robin-Neumann conditions with $\beta=0.1$ : the initial configuration is $m(x, y)=1$ for $\{(x, y) \mid x<0.25+0.15 \sin (4 \pi y)\}$ and $m(x, y)=-1$ otherwise. The configurations of $m(x, y)$ at iterations 0,1 , 3 , and 23 and the corresponding principal eigenvalue at different iterations.

\section{REFERENCES}

[1] G. A. Afrouzi. Boundedness and monotonicity of principal eigenvalues for boundary value problems with indefinite weight functions. Int. J. Math. Math. Sci., 30(1):25-29, 2002.

[2] G. A. Afrouzi and K. J. Brown. On principal eigenvalues for boundary value problems with indefinite weight and Robin boundary conditions. Proc. Amer. Math. Soc., 127(1):125-130, 1999.

[3] W. E. Arnoldi. The principle of minimized iteration in the solution of the matrix eigenvalue problem. Quart. Appl. Math., 9:17-29, 1951.

[4] F. Belgacem. Elliptic Boundary Value Problems with Indefinite Weights: Variational Formulations of the Principal Eigenvalue and Applications, Pitman Research Notes in Mathematics, Vol. 368. Longman, Harlow, U.K., 1997.

[5] M. Bôcher. The smallest characteristic numbers in a certain exceptional case. Bull. Amer. Math. Soc., 21(1):6-9, 1914.

[6] R. S. Cantrell and C. Cosner. Diffusive logistic equations with indefinite weights: population models in disrupted environments. Proc. Roy. Soc. Edinburgh Sect. A, 112(3-4):293-318, 1989.

[7] R. S. Cantrell and C. Cosner. Diffusive logistic equations with indefinite weights: population models in disrupted environments. II. SIAM J. Math. Anal., 22(4):1043-1064, 1991. 

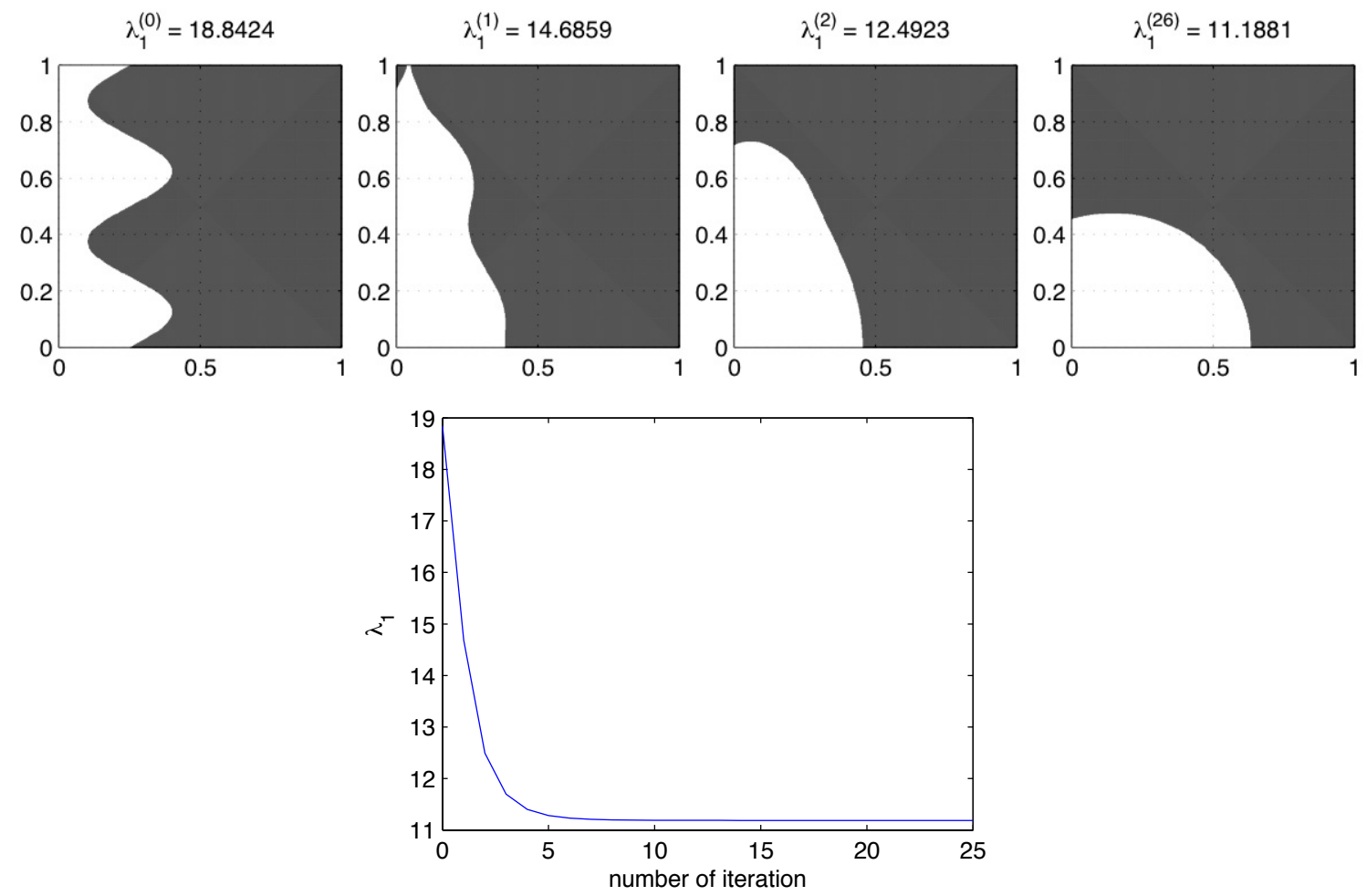

FiguRE 8. Mixed Robin-Neumann conditions with $\beta=1$ : the initial configuration is $m(x, y)=1$ for $\{(x, y) \mid x<0.25+0.15 \sin (4 \pi y)\}$ and $m(x, y)=-1$ otherwise. The configurations of $m(x, y)$ at iterations $0,1,2$, and 26 and the corresponding principal eigenvalue at different iterations.

[8] R. S. Cantrell and C. Cosner. The effects of spatial heterogeneity in population dynamics. J. Math. Biol., 29(4):315-338, 1991.

[9] R. S. Cantrell and C. Cosner. Spatial ecology via reaction-diffusion equations. Wiley Series in Mathematical and Computational Biology. John Wiley \& Sons Ltd., Chichester, 2003.

[10] Carlos Conca, Rajesh Mahadevan, and Leon Sanz. Shape derivative for a two-phase eigenvalue problem and optimal configurations in a ball. In CANUM 2008, volume 27 of ESAIM Proc., pages 311-321. EDP Sci., Les Ulis, 2009.

[11] S. Cox. The two phase drum with the deepest bass note. Japan J. Indust. Appl. Math., 8:345-355, 1991.

[12] S. Cox and J. McLaughlin. Extremal eigenvalue problems for composite membranes i and ii. Applied Math and Optimization, 22:153-167, 1990.

[13] W. H. Fleming. A selection-migration model in population genetics. J. Math. Biol., 2(3):219-233, 1975.

[14] P. Grisvard. Elliptic problems in nonsmooth domains, volume 24 of Monographs and Studies in Mathematics. Pitman (Advanced Publishing Program), Boston, MA, 1985.

[15] L. He, C.-Y. Kao, and S. Osher. Incorporating topological derivatives into shape derivatives based level set methods. J. Comp. Phys., 225:891-909, 2007.

[16] P. Hess and T. Kato. On some linear and nonlinear eigenvalue problems with an indefinite weight function. Comm. Partial Differential Equations, 5(10):999-1030, 1980. 

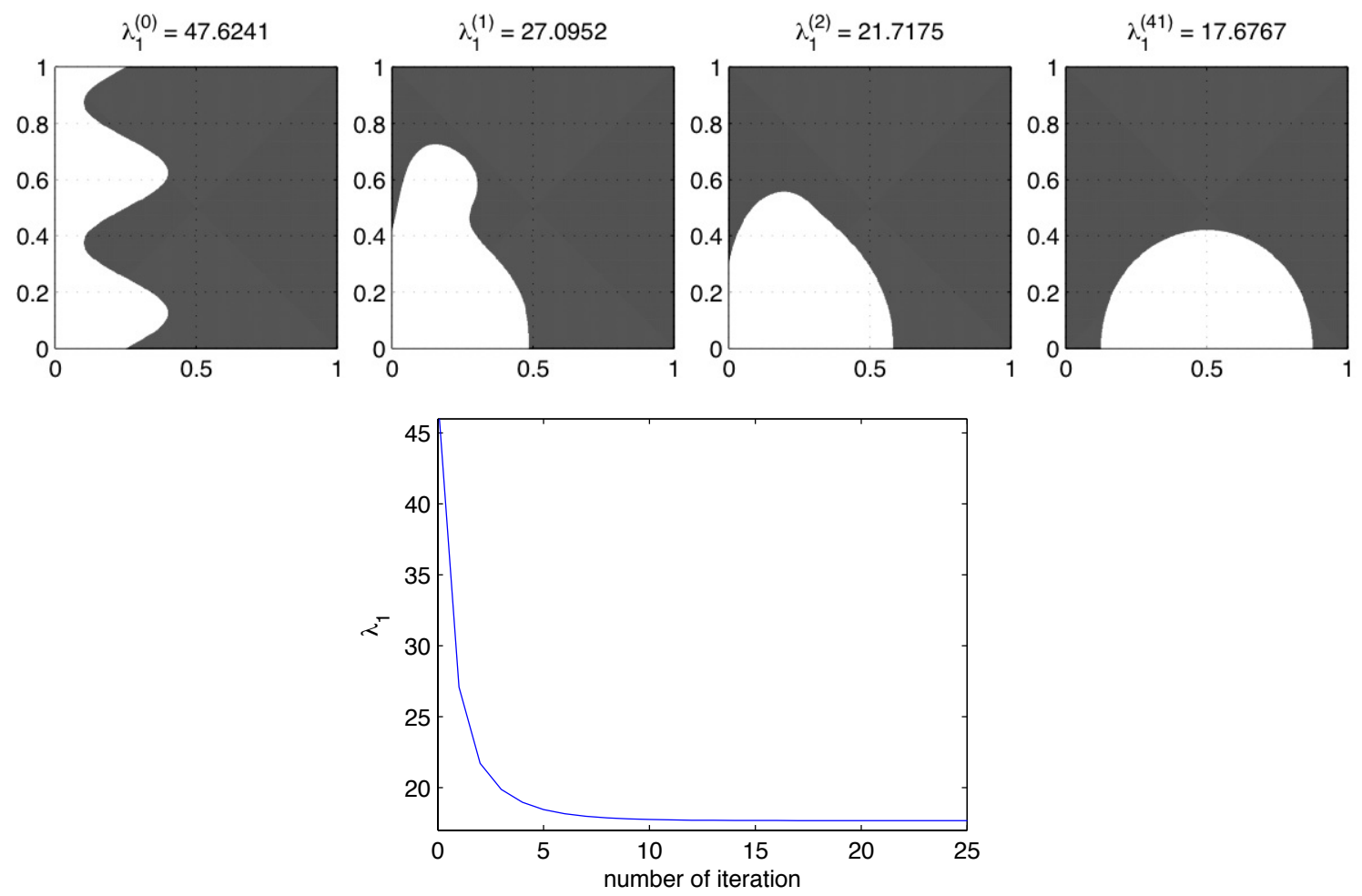

FiguRE 9. Mixed Robin-Neumann conditions with $\beta=10$ : the initial configuration is $m(x, y)=1$ for $\{(x, y) \mid x<0.25+0.15 \sin (4 \pi y)\}$ and $m(x, y)=-1$ otherwise. The configurations of $m(x, y)$ at iterations $0,1,2$, and 41 and the corresponding principal eigenvalue at different iterations.

[17] C.-Y. Kao, Y. Lou, and E. Yanagida. Principal eigenvalue for an elliptic problem with indefinite weight on cylindrical domains. Math Biosci Eng, 5(2):315-35, 2008.

[18] C.Y. Kao and S. Su. An efficient rearrangement algorithm for shape optimization on eigenvalue problems. IMA workshop on Numerical Solutions of Partial Differential Equations: Fast Solution Techniques, 2010.

[19] M. G. Krein. On certain problems on the maximum and minimum of characteristic values and on the Lyapunov zones of stability. Amer. Math. Soc. Transl. (2), 1:163-187, 1955.

[20] Y. Lou and E. Yanagida. Minimization of the principal eigenvalue for an elliptic boundary value problem with indefinite weight, and applications to population dynamics. Japan J. Indust. Appl. Math., 23(3):275-292, 2006.

[21] J. Osher and F. Santosa. Level set methods for optimization problems involving geometry and constraints i. frequencies of a two-density inhomogeneous drum. Journal of Computational Physics, 171:272$288,2001$.

[22] J.-C. Saut and B. Scheurer. Remarks on a non-linear equation arising in population genetics. Comm. Partial Differential Equations, 3(10):907-931, 1978.

[23] J. G. Skellam. Random dispersal in theoretical populations. Biometrika, 38:196-218, 1951. 


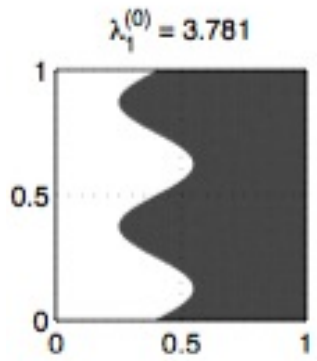

$\left(a_{1}\right)$

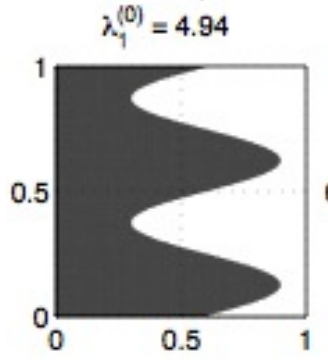

$\left(c_{1}\right)$

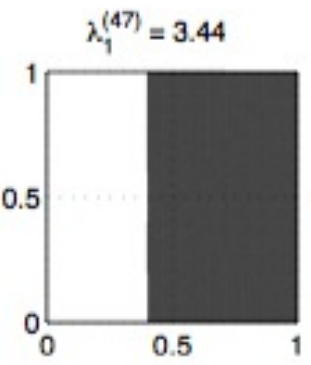

$\left(a_{2}\right)$

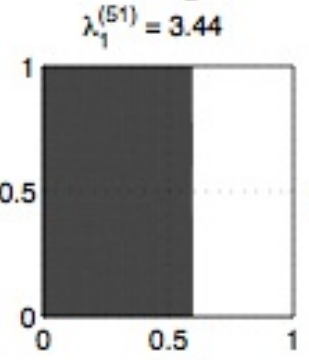

$\left(\mathrm{C}_{2}\right)$

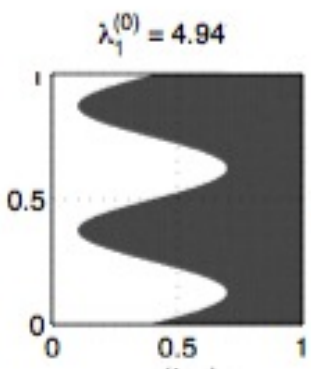

$\left(b_{1}\right)$

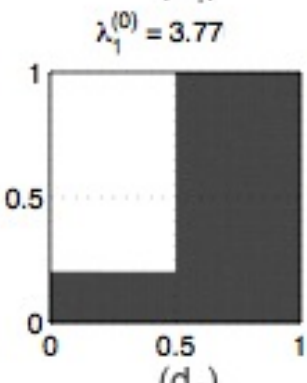

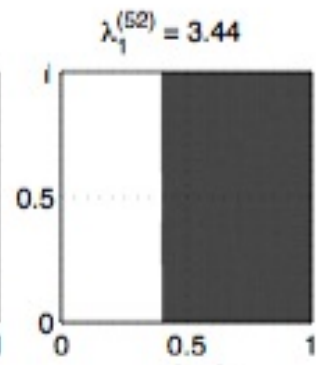

$\left(b_{2}\right)$

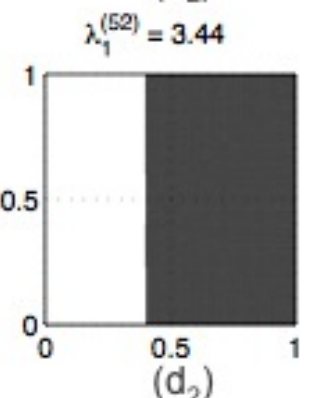

Figure 10. Mixed Robin-Neumann conditions with $\beta=0.1$ : the initial configurations are $\left(a_{1}\right) m(x, y)=1$ for $\{(x, y) \mid x<0.4+0.15 \sin (4 \pi y)\}$ and $m(x, y)=-1$ otherwise, $\left(b_{1}\right) m(x, y)=1$ for $\{(x, y) \mid x<0.4+0.3 \sin (4 \pi y)\}$ and $m(x, y)=-1$ otherwise, $\left(c_{1}\right) m(x, y)=1$ for $\{(x, y) \mid x>0.6+$ $0.3 \sin (4 \pi y)\}$ and $m(x, y)=-1$ otherwise, and $\left(d_{1}\right) m(x, y)=1$ for $\{(x, y) \mid x<0.5, y>0.2\}$ and $m(x, y)=-1$ otherwise. The configurations of optimal $m(x, y)$ are shown in $\left(a_{2}\right),\left(b_{2}\right),\left(c_{2}\right)$, and $\left(d_{2}\right)$.

[24] J. Sokołowski and A. Żochowski. On the topological derivative in shape optimization. SIAM J. Control Optim., 37(4):1251-1272 (electronic), 1999.

[25] Jan Sokołowski and Jean-Paul Zolésio. Introduction to shape optimization, volume 16 of Springer Series in Computational Mathematics. Springer-Verlag, Berlin, 1992. Shape sensitivity analysis.

[26] S. Su. Numerical approaches on shape optimization of elliptic eigenvlaue problems and shape study of human brains. Ph.D thesis, The Ohio State University, 2010.

[27] A. Wayne. Inequalities and inversions of order. Scripta Mathematica, 12(2):164-169, 1946.

Humboldt-University of Berlin, Unter den Linden 6, 10099 Berlin, Germany and Institute of Mathematics and Scientific Computing, University of Graz, Heinrichstrasse 36, A-8010 Graz, Austria. E-mail: hint@math.hu-Berlin.de

Department of Mathematics, The Ohio State University, 410 Math Tower, 231 West 18th Avenue, Columbus, OH 43210-1174. U.S.A. E-mail: KaO@math.ohiO-STate.edu

Department of Mathematics, Johann-von-Neumann-Haus, Rudower Chaussee 25, D-12489 Berlin-Adlershof, Germany. E-mail: laurain@math.hu-Berlin.de 

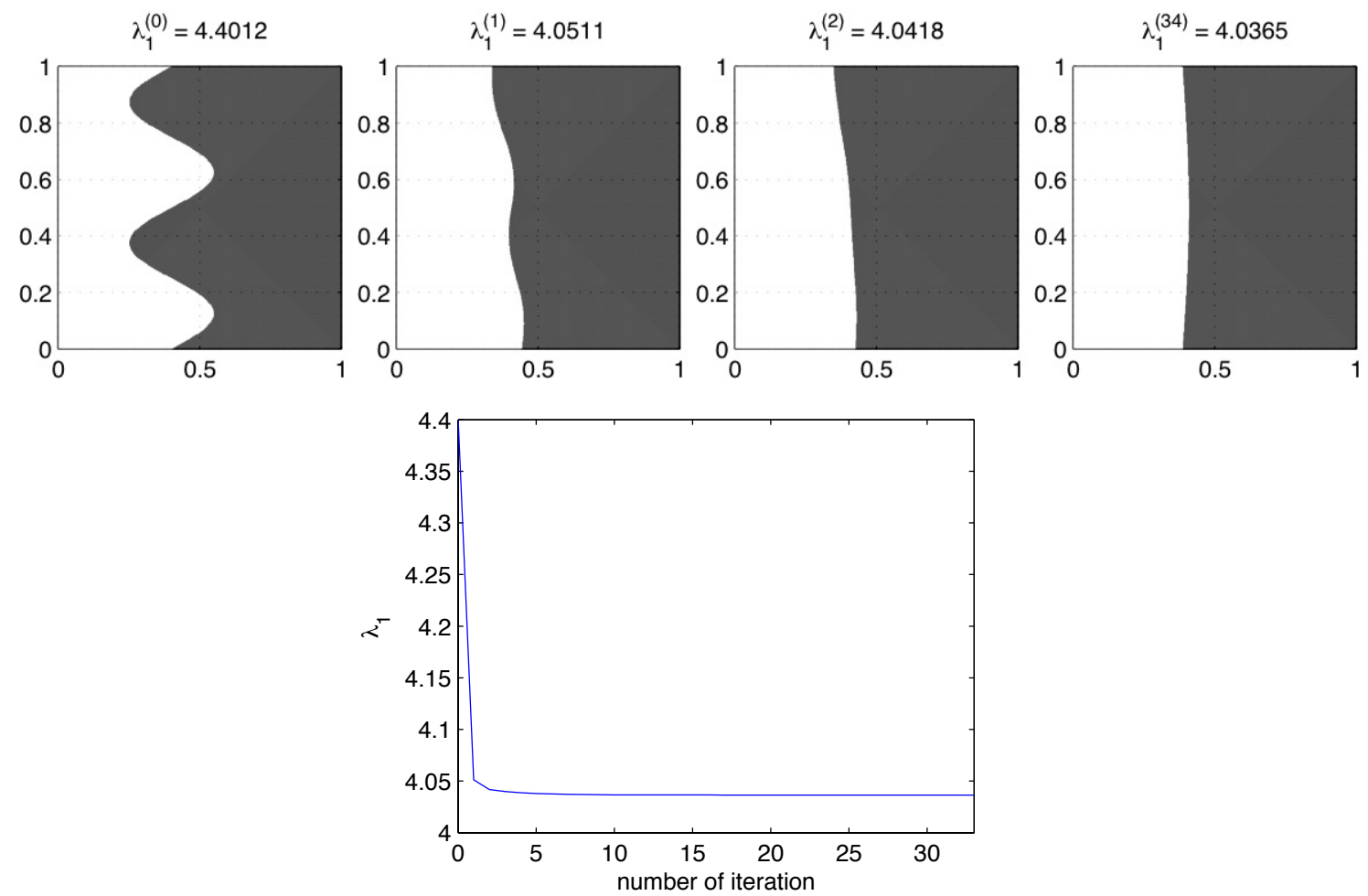

FIgURE 11. Robin boundary condition with $\beta=0.1$ : the initial configuration is $m(x, y)=1$ for $\{(x, y) \mid x<0.4+0.15 \sin (4 \pi y)\}$ and $m(x, y)=-1$ otherwise. The configurations of $m(x, y)$ at iterations $0,1,2$, and 34 and the corresponding principal eigenvalue at different iterations. 

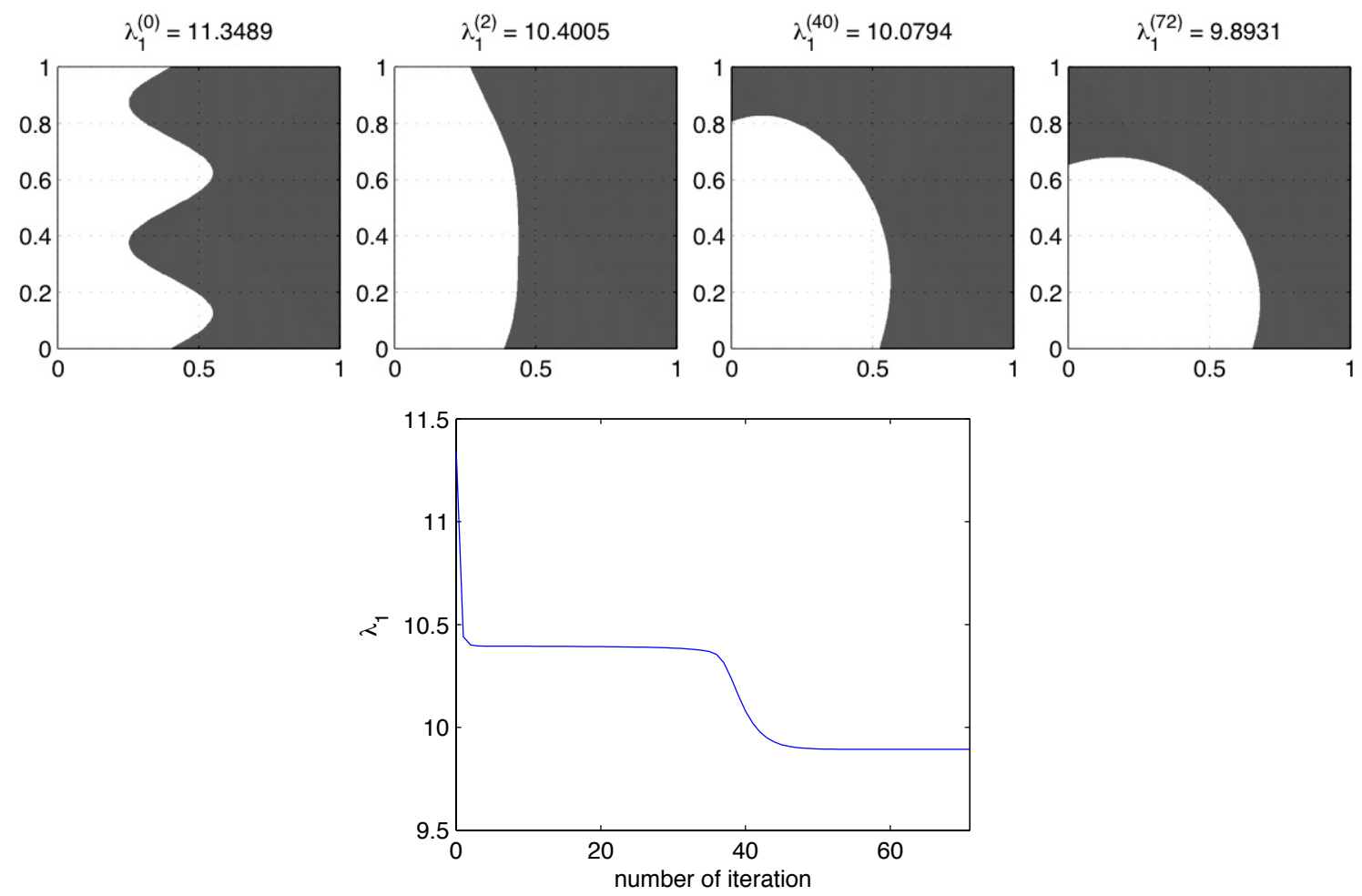

FIGURE 12. Robin boundary condition with $\beta=1$ : the initial configuration is $m(x, y)=1$ for $\{(x, y) \mid x<0.4+0.15 \sin (4 \pi y)\}$ and $m(x, y)=-1$ otherwise. The configurations of $m(x, y)$ at iterations $0,2,40$, and 72 and the corresponding principal eigenvalue at different iterations. 

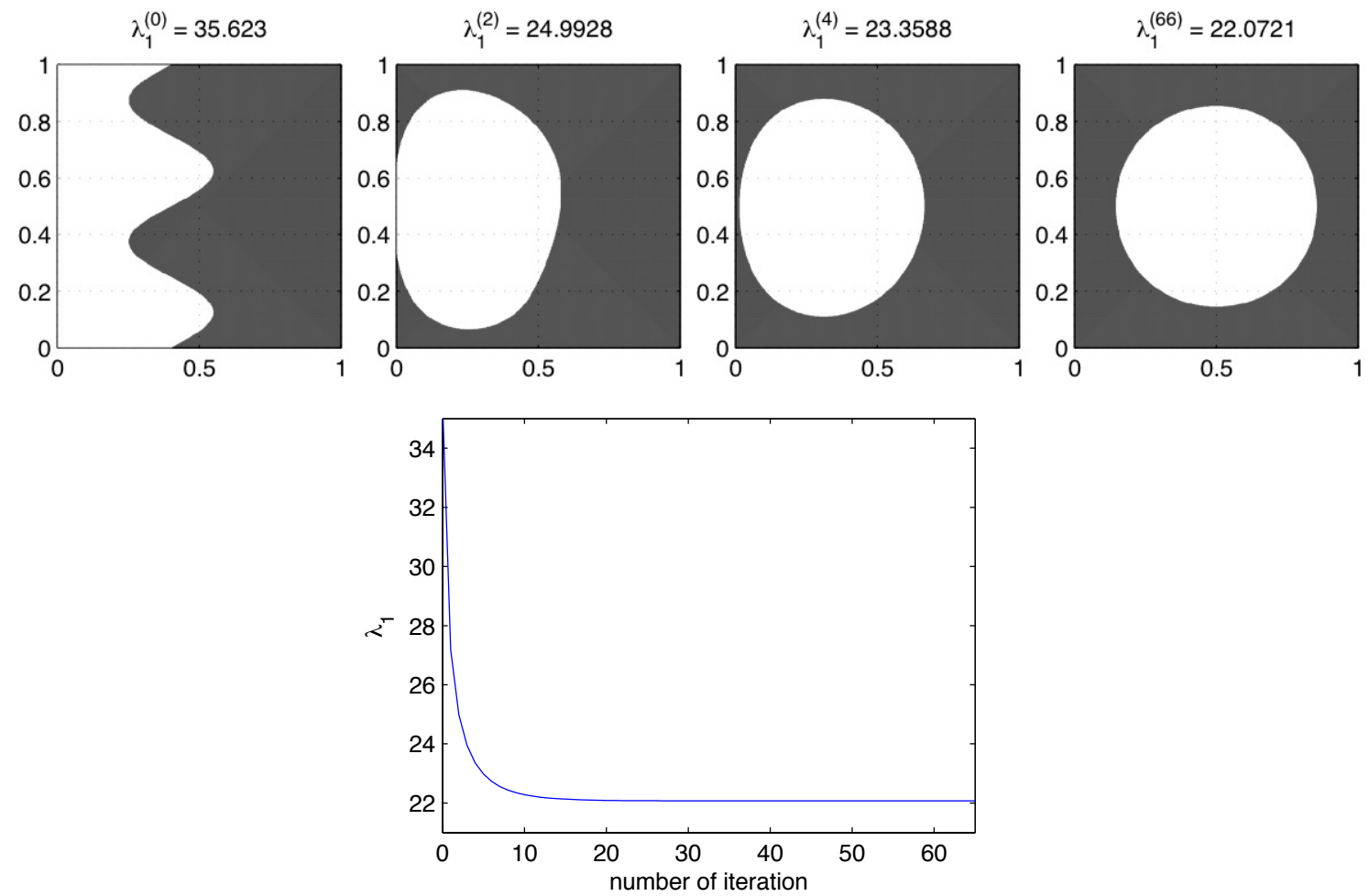

FIgURE 13. Robin boundary condition with $\beta=10$ : the initial configuration is $m(x, y)=1$ for $\{(x, y) \mid x<0.4+0.15 \sin (4 \pi y)\}$ and $m(x, y)=-1$ otherwise. The configurations of $m(x, y)$ at iterations $0,2,4$, and 66 and the corresponding principal eigenvalue at different iterations. 